\title{
Welchen Zweck verfolgt das Schwefeln und Talken der Graupen?
}

\author{
Von \\ W. Plücker und R. Flebbe. \\ Berichterstatter: W. Plücker, Solingen.
}

Infolge des bekannten vom Kaiserlichen Gesundheitsamt seinerzeit herbeigeführten Erlasses gegen das Talken und Schwefeln der Graupen wandte sich der Verein Deutscher Graupenmüller in einer Denkschrift ${ }^{1}$ ) an den Reichskanzler, deren wesentliche Sätze hier mitgeteilt werden sollen:

„In den Kerben des Graupenkornes, begünstigt von den mehligen Bestandteilen der meistens künstlichen Umhüllung bildeten sich besonders im Sommer unzählige mikroskopisch kleine Tiere, die, äußerlich unerkennbar, nicht nur den Geschmack beeinträchtigten, sondern auch häufig einen beschleunigten Zersetzungsprozeß hervorriefen. Da die frühere Herstellungsweise selbst somit kein Mittel bot, dem Übelstande abzuhelfen, im Gegenteil diesen Übelstand direkt begünstigte, so versuchte man, angeregt durch die Erfolge bei anderen, leicht dern Verderben ausgesetzten Nahrungsmitteln, wie Dörrobst und dergleichen, zunächst in Holland und England die Graupenfabrikate bezw. die Rohstoffe mit stark verdünter schwefliger Säure zu behandeln, um die etwa vorhandenen Milben oder die ihre Existenz begünstigenden Bestandteile zu vernichten und so die Fabrikate zu sterilisieren.

Um die leichten bolzfaserigen Teilchen der Gerstenhülse, welche von den Roll. gängen nur teilweise gelöst, aber nicht entfernt werden, vom Graupenkorn zu beseitigen, hat man seit längerer Zeit ein Polierverfahren angewendet, indem man die Graupen mit Magnesiumsilicat (Talkummehl) in Reibung bringt und das letztere nachher mit starker Luftströmung wieder absaugt. Durch dieses auch bei der Reisfabrikation seit Jahren angewendete Verfahren wird der Graupe eine viel sauberere Beschaffenheit verliehen und ibre Haltbarkeit vermehrt, da selbst die verschwindend kleimen Mengen, welche am Graupenkorn haften bleiben, die Graupe gegen Witterungseinflüsse schützen und $\mathrm{ibr}$ eine feine Glätte verleihen, welche die Bildung never schädlicher Mehlschichten, wie sie z. B. während des Transportes infolge der Reibung des runden Kornes unvermeidlich sein würde, verhindert.

Erst durch die zuerst von den holländisehen und englisehen Graupenmühlen entdeckte Möglichkeit dex besseren Konservierung konnte auch in Deutschland die Graupenfabrikation in größerem Maßstabe aufgenommen und so dem Konsum neben einem guten wohlschmeckenden und außerordentlich nahrhaften auch ein gut zu konservierendes, billiges Nabrungsmittel geliefert werden.

Das Gutachten des Reichsgesundheitsamtes kann nur auf unrichtige Information und auf darauf beruhende Voraussetzungen zurückzuführen sein, die speziell bei der Graupenfabrikation ganz unmöglich als vorhanden angenommen werden können, denn es handelt sich hier keineswegs um eine Verfälschung, sondern um eine Vermehrung der Haltbarkeit, also um eine entschiedene Verbesserung eines Nahrungsmittels, und es handelt sich auch nicht um eine Täuschung des kaufenden Publikums, denn dieses

1) Zeitschr. f. offentl. Chem. 1905, 11, 217. 
verlangt eine saubere, gegen eine schnelle Zersetzung und gegen Milbenbildung geschützte lager- und versandfähige Handelsware.

Die ergebenst Unterzeichneten erkennen selbstverständlich die Notwendigkeit an, daß die Anwendung der vorbenannten Konservierungsmittel hinsichtlich der zu verwendenden Quantitäten, wie z. B. auch bei Dörrobst, in gewissen Schranken zu halten ist, daf z. B. nach dem Polierverfahren das Talkum soweit zu entfernen wäre, als dieses technisch mit den vorhandenen Mitteln möglich, wobei man aber mit der Tatsache rechnen muß, daß sich hauptsächlich bei grobkörnigen Graupen, ohne daß solches zu verhindern wäre, in den Einschnitten des Kornes Talkumteilchen festsetzen, die nicht wieder zu entfernen sind. Seine Exzellenz der Herr Reichskanzler Graf von Bülow wolle unter Berücksichtigung der vorhin entwickelten Umstände und nach Anhörung des Kaiserlichen Gesundbeitsrates, die wir dringend erbitten, veranlassen, daß die Anwendung von schwefliger Säure und Talkumpulver (Magnesiumsilikat) zwecks Vermehrung der Haltbarkeit der Graupen, Grütze und deren Nebenfabrikaten insoweit gestattet werde, als die den Fabrikaten zugeführten Mengen eine Konservierung und Polierung bezwecken und den Gehalt an schwefliger Säure von $0,125 \%$ und an Talkum von $0,6 \%$ nicht überschreiten".

Das Kaiserliche Gesundheitsamt trat darauf in eine erneute Prüfung der Angelegenheit ein; das Ergebnis dieser Versuche war folgendes: „Die bei der Herstellung von Graupen vielfach als Ausgangsmaterial verwendete, ausländische, vornehmlich russische Futtergerste kann, sofern gesunde Ware verarbeitet wird, nicht als ungeeignet oder gegenüber inländischer Gerste als minderwertig angesehen werden.

Das Behandeln der zur Herstellung von Graupen dienenden Gerste mit schwefliger Säure ist hygienisch unbedenklich, wenn sich die Mengen schwefliger Säure im fertigen Erzeugnisse in den bisher beobachteten niedrigen Grenzen (nicht mebr als $20 \mathrm{mg}$ auf $100 \mathrm{~g}$ verkaufsfertiger Ware) halten. Die Verwendung von Talkum als Gleit- und Poliermittel ist entbehrlich and unerwünscht. Als hygienisch gleichgültig kann sie jedoch geduldet werden, wenn

a) der Gebrauch auf das Mindestmaß beschränkt bleibt, so zwar, daß nur solche Mengen an der fertigen Ware verbleiben, die sich bei den in der Technik gebräuchlichen Verfahren nicht vermeiden lassen (vergl. die ähnliche Vorschrift im $\$ 10$ des Gesetzes, betr. die Verwendung gesundheitsschädlicher Farben vom 5. Juli 1887, Reichs-Gesetzblatt S. 277), und wenn

b) der Zusatz nicht zur Täuschung im Handel und Verkehr benutzt wird. (Vergl. \$ 10 des Gesetzes, betr. den Verkehr mit Nahrungsmitteln usw, vom 14. Mai 1879, Reichs-Gesetzblatt Seite 145.)"

Wegen eines gerichtlichen Verfahrens haben wir uns veranlaßt gesehen festzustellen, ob die Angaben der Graupenmüller bezüglich der Konservierung zutreffend sind oder nicht, da diese Frage in obigem Ergebnis des Kaiserlichen Gesundheitsantes nicht berührt worden ist. Ehe wir auf unsere diesbezüglichen Untersuchungen eingehen, dürfte es zweckmäßig sein, kurz auf die Zersetzung der hier in Frage kommenden Nahrungsmittel durch Kleinlebewesen einzugehen.

Der Keimgehalt ist von verschiedenen Bedingungen abhängig, $z, B$. von dem Bau der Samen; solche mit behaarter oder rauher Oberfläche werden mehr Keime aufweisen als solche mit glatter. Auch das Wetter während der Entwickelung und der Ernte ist von Einfluß. Während der Blüte der Gerste siedeln sich auf den zu jener Zeit sebr zuckerreichen behaarten Schüppchen Bakterien usw. an, ebenso auf 
der Narbe, und es bilden sich die von Lindne ${ }^{1}$ ) und Chrzaczsz ${ }^{2}$ ) beschriebenen Schüppchenvegetationen, die aus Bakterien, Hefe, Schimmelpilzen, Brandsporen usw. bestehen. Richter ${ }^{3}$ ) fand unter beregneter Gerste $15 \%$, unter nicht beregneter $3 \%$ braunspitzig und Reichard4) erwähnt beregnete Gerste, die ganz mit BakterienZooglöen überzogen war. Solche Zooglöen bildende Bakterien sind namentlich Bact. fluorescens liquefac. und Bac. herbicola aureus.

Nach den Versuchen von Hoffman $n^{5}$ ) handelt es sich bei dem Reichtum an Bakterien usw, die unsere Samen und Früchte aufweisen, nicht nur um angeflogene Keime, sondern um eine eigene Pilzflora, die nach $B u r^{6}{ }^{6}$ ) und $D$ üggeli ${ }^{7}$ ) von der Bodenflora verschieden ist. Vornehmlich findet man von Bakterien: Bact. fluorescens liquefaciens, Bac. herbicola aureus, Bact putidum, coli commune, Bac. mesentericus vulgatus, Milch- und Buttersäurebakterien, Pektinvergärer usw. Globi ${ }^{8}$ ) untersuchte eine größere Anzahl thermophiler Bakterien, darunter auch thermotolerante, die sowohl bei 15 wie bei $68^{0}$ wuchsen, und Rabinowitsch ${ }^{9}$ ) konnte die weite Verbreitung solcher Arten in Dünger, Getreide, Milch usw, nachweisen. Von Bakterien, die bei einer Infektion auftreten, kommt ein von $\mathrm{Hiltn}$ e ${ }^{10}$ ) in Lupinensamen öfter aufgefundenès anaerobes, pektinvergärendes Stäbchen in Betracht. Gesunde Samen enthalten in ihrem Innern im allgemeinen keine Pilze, die gegenteiligen Angaben von Bernheim ${ }^{11}$ ) sind nach Buchner ${ }^{12}$ ), Lehmann ${ }^{13}$ ), Hiltner ${ }^{10}$, auf fehlerhafte Untersuchungsmethoden zurückzuführen. Saprophytische Pilze siedeln sich im Innern der Samen nur an, wenn dieselben in reifen Zustande aus irgend welchen Gründen längere Zeit feucht gehalten wurden. Von Eumyceten kommen hierbei nach Hiltne ${ }^{10}$ ) hauptsächlich die gewöhnlichen Schimmelpilze in Betracht. Bei der Gerste tritt manchmal eine Infektion durch Ustillago hordei ein in der Weise, daß während der Blütezeit ausitäubende Brandsporen die Narbe infizieren und, wie $\mathrm{Hecke}^{\mathbf{1 4}}$ ) nachwies, den Embryo durchwuchern. Auch Helmin thosporium kommt innerhalb der Cerealien-Körner vor, wie $R$ emer ${ }^{15}$ ) und $R$ avn ${ }^{16}$ ) feststellten. Letzterer fand in zablreichen Gerstenproben Helminthosporium teres. Bezüglich Fusarium sei auf die Arbeit von Klein ${ }^{17}$ ) verwiesen. Vorwiegend kommt es auf der Außenseite der Spelze vor, einzelne Hyphen dringen aber auch ins Innere des Gerstenkorns und färben die Stärke rot. Tsik-

1) Lindner, Mikrosk. Betriebskontrolle im Gärungsgewerbe 1905, S. 107.

2) Wochenschr. f. Brauerei 1902, 19, 590.

3) Allg. Braner- und Hopfenzeitung 1891, 7, 67.

4) Chem.-Ztg. 1897, 21, 21 .

5) Wochenschr. f. Brauerei 1896, 18, 1153.

8) Zentralbl. f. Bakteriol. II. Abt. 1903, 10, 756.

7) Zentralbl. f. Bakteriol. II. Abt, 1904, 12, 602 and 1904, 13, 56.

8) Zeitschr. f. Hygiene 1888, 3, 294.

9) Zeitschr. f. Hygiene 1895, 20, 154.

10) Arb. aus der biol. Abt. d. Kaiserl. Ges.-Amtes 1902, 3, 1.

$\left.{ }^{11}\right)$ Münchener mediz. Wochenschr. 1888, 35, 743.

$\left.{ }^{12}\right)$ Zentralbl. f. Bakteriol. $1889,5,341$.

${ }^{13}$ ) Archiv f. Hygiene 1889, 9, 350.

11) Ber. d. deutsch. botan. Gesellseh. 1905, 23, 248.

15) 80. Jahresber. d. Schles. Gesellschaft f. vater]. Kultur, Breslau 1905, II. Abt. 22.

16) Zeitschr. f. Pflanzenkrankheiten 1901, 11, 1.

17) Der Bierbrauer 1892, 23, 1247. 
linsky ${ }^{1}$ ) beschrieb einen als Thermo-actinomyces vulgaris bezeichneten Pilz, der zwischen 42 und $46^{\circ} \mathrm{C}$ wuchs und auf Getreide und Kartoffeln sehr verbreitet ist.

Die Aufbewahrung ist natürlich auch von großem Einflub. Hoffmann fand bei einer Gerste aus der Umgegend von Berlin 4032000 Keime in 1 g. Eine andere Probe sehr guter Gerste hatte noch 200000 Keime ${ }^{2}$ ). Durch trockene Lagerung wird der Keimgehalt vermindert. So sank derselbe bei der zuletzt erwähnten Gerste mit 200000 Keimen im g nach 3-monatlicher Aufbewahrung in einer Blechbüchse auf 1120. Die Zahl der Schimmelpilze hatte sich im Gegensatz zu den Bakterien nicht oder nur wenig vermindert.

Lüften und häufiges Umschaufeln wirken nach Hoffmann ${ }^{3}$ ) ähnlich. Bei zerquetschten Körnern steigt der Keimgehalt ins Ungeheure. Die rote Färbung mancher Körner rührt nach $\mathrm{Will}^{4}$ ) von roter Hefe her, die das Korn fast ganz einhüllt. Erhöhte Temperatur und Feuchtigkeit müssen den Keimgehalt stark vermehren. Nach Hoffmann ${ }^{5}$ ) hängt der Wassergehalt des Getreides im wesentlichen vom Feuchtigkeitsgehalt der Luft ab. Die Atmung und dadurch bedingte Wasserbildung bilden eine Ursache möglicher Wasseranreicherung in lagerfestem Getreide. Als Ursache des Warmwerdens kommt die Eigenatmung des Getreides und der auf seiner Oberfläche sitzenden Mikroben in Betracht, die um so lebhafter sein wird, je feuchter das Getreide ist. Die Atmungstätigkeit ist bei lagerfestem Getreide (nach Schulze ist Gerste mit einem Wassergehalt von $12 \%$ durchaus lagerfest) sehr gering. Kolkwitz ${ }^{6}$ ) stellte fest, daß $1 \mathrm{~kg}$ Gerste mit einem Wassergehalt von $10-15 \%$ in 24 Stunden $0,3-1,5 \mathrm{mg}$ Kohlensäure bildet. Nimmt der Wassergehalt zu, so steigt die Atmungstätigkeit sehr schnell, bei einem Wassergehalt von $20,5 \%$ entwickelte $1 \mathrm{~kg}$ Gerste in 24 Stunden $359 \mathrm{mg}$ Kohlensäure, bei $33 \%$ Wasser sogar 2,0 g Kohlensäure.

Zerkleinertes Getreide atmet viel stärker. So erzeugt Mehl und Schrot bei gleichem Wassergehalt mehr Kohlensäure als das unverletzte Getreide. Ebenso wie das Getreide selbst werden natürlich die auf der Oberfläche sitzenden Kleinlebewesen Kohlensäure erzeugen.

Notwendig für die Entwickelung der Mikroben ist vor allen Dingen ein gewisser Feuchtigkeitsgehalt. Bei niedrigem Wassergehalt, bei dem sich nur Eumyceten entwickeln können, ist Sauerstoff notwendig. Bei höherem Wassergehalt, der auch die Vermehrung von Schizomyceten gestattet, ist Sauerstoff nicht mehr vonnöten. Von großer Bedeutmng für die Entwickelung der Mikroben ist auch die Struktur. Mehl wird infolge seiner Feinheit viel schneller Wasser absorbieren als das ganze Getreide, infolgedessen verdirbt es auch schneller. Was den Wassergehalt anbetrifft, so bedürfen Pilze eines solchen von 14-18\% und mehr. Hellriegel ${ }^{7}$ ) gibt für Schnitzel, Raps und Erdnußmehl 14-18\%, Spieckermann und Bremer ${ }^{8}$ ) geben für Baumwollsaatmehl $14-15 \%$ und König, Spieckermann und Kutten.

1) Annal. Pasteur 1899, 13, 788.

9) Hoffmann, Das Versuchskornhaus, Berlin 1904, S. 204.

3) Wochenschr, f. Brauerei 1896, 13, 1153.

4) Zeitschr. f. d. ges. Brauwesen 1904, 27, 521.

5) Wochenschr. f. Braverei 1905, 22, 80 u. 249.

9) Ber. d. dentsch. botan. Gesellachaft 1901, 19, 285. - Blätter für Gersten-, Hopfenund Kartoffelbau 1901, 3, 370.

7) Biedermann's Zentralbl, 1885, 14, 93.

8) Landwirtsch. Jahrbücher 1902, 31, 88. 
keuler ${ }^{1}$ für Sesammehl dieselben Zahlen als untere Grenze an. Spieckermann und Breme $\mathbf{r}^{2}$ ) haben bei Baumwollsaatmehl beobachtet, welche Organismen sich bei verschiedenem Wassergehalt entwickeln. Bei 14-15\% waren es Aspergillus glaucus, Aspergillus repens, Eurotium rubrum, bei $20 \%$ Monilia-Arten, bei $23 \%$ Penicillium. Auch $\mathrm{Haselh}$ off und $\mathrm{Mach}^{3}$ ) stellten fest, daß z. B. Apergillus oryzae und Penicillium glaucum verschieden hohe Ansprüche an den Wassergehalt stellen. Unter gewöhnlichen Verhältnissen verlaufen die Zersetzungen bei mittlerer Temperatur. Infolgedessen vermehren sich nur die hierbei am besten gedeihenden Arten. Tritt dagegen infolge Selbsterhitzung eine Temperaturerhöhung ein, so beobachtet man z. B. Aspergillus flavus, Rhizopus nigricans und andere, die unter gewöhnlichen Bedingungen nicht vorkommen. Eumyceten stellen geringere Anforderungen an den Nährboden wie Schizomyceten. Bei hohem Gehalt an Kohlenhydraten, wie bei Mehlen, Melassen usw., entwickeln sich bei Luftabschluß kohlenhydratvergärende Organismen, wie Milchsäurebakterien, Bact. coli, aerogenes usw., bei Luftzutritt on der Oberfläche namentlich Kartoffelbacillen sowie Eumyceten. Letztere verbrennen zum Teil die durch Gärung entstandenen Säuren, zum Teil neutralisieren sie dieselben durch Ausscheidung alkalischer Produkte. Ist dies der Fall, so kommen auch die obligaten Anaerobier und Bac. putrificus usw. zur Entwickelung. Sind dagegen nur geringe Mengen von Kohlenhydraten vorhanden und reichlich Proteinstoffe, wie z. B. in den Fleischmehlen, so beginnt sowohl bei Luftzutritt wie bei LuftabschluB sehr bald die Zersetzung der Proteinstoffe. Schizomyceten, welche zur Zersetzung des Baumwollsaatmehles und ähnlich zusammengesetzter Rückstände der Ölgewinnung beitragen, sind sowohl Kohlenhydrate wie Protein vergärende Organismen ${ }^{4}$ ).

Wie sehr der Wassergehalt die Bakterientätigkeit begünstigt, ergibt sich aus einer Arbeit von König, Spieckermann und Tillmans ${ }^{5}$ ). Sie verrieben Roggenmehl, welches $10 \%$ Wasser enthielt, mit so viel Wasser, daß der Gehalt hieran auf 20,25 und $30^{\%} \%$ anwuchs, und bewahrten bei 11 und $18^{\circ}$ auf. Nach 8 Tagen waren die bei $18^{\circ}$ aufbewahrten Proben mit 25 und $30 \%$ Wasser stark verschimmelt, von den bei $11^{\circ}$ aufbewahrten nur die mit $30 \%$. Die Proben wurden darauf bei $75^{\circ}$ sterilisiert und die Anzahl der hitzebeständigen Keime bestimmt.

$\begin{array}{lccc}\text { Wassergehalt: } & 20 & 25 & 30 \% \\ \text { Aufbewahrungs:Temperatur } 11^{\circ} & 13105 & 32780 & 146750 \\ \text { Temperatur 18 } & \text { Unzählbar } & \text { Unzäblbar } & \text { Unzählbar }\end{array}$

Vorber enthielt das Mehl nur 800 Keime in $1 \mathrm{~g}$ und darunter 110 kochfeste. Ein Umstand, der die Entwickelung der Kleinlebewesen auch sehr begünstigt, ist das öfters auftretende Schwitzen des Getreides. Schwitzen des Getreides findet statt, wenn es in abgekühltem Zustande mit warmer feuchter Luft zusammentrifft. Tierische Schmarotzer fördern ebenfalls die Entwickelung der Kleinlebewesen, indem durch ihre Ausscheidungsprodukte und deren ammoniakalische Zersetzung ein günstiger Entwickelungsboden für manche Pilze, z. B. Aspergillus albus usw., entsteht.

1) Diese Zeitschrift 1906, 11, 178.

2) Landwirtsch. Jahrbücher 1902, 31, 88.

3) Land wirtschaftl. Jahrbücher 1906, 35, 445.

4) Vgl. König, Spieckermann u. Olig, diese Zeitschrift 1903, 6, 193, sowie König, Spieckermann u. Kuttenkeuler, diese Zeitschrift 1906, 11, 178.

5) Diese Zeitschrift 1902, 5, 763, 
Ähnlich wie die Entwickelung der Bakterien und Pilze ist auch diejenige der Milben an einen bestimmten Wassergehalt gebunden, wie die Untersuchungen von Maurizio ${ }^{1}$ ) beweisen. In Heu und Häcksel mit 7-9,2\% Wasser vermehrten sie sich gar nicht, wohl aber, wenn der Wassergehalt 13,5-14\% betrug. Ebenso trat bei Mehlen mit $6-8 \%$ Wasser keine Entwickelung ein, aber lebhafte Vermehrung, sobald der Wassergebalt auf $15-16 \%$ stieg. Die nachfolgende Tabelle von Maurizio gibt hierüber am besten Aufschluß.

Vermehrung der Milben in Futterstoffen bei verschiedenem Wassergehalte.

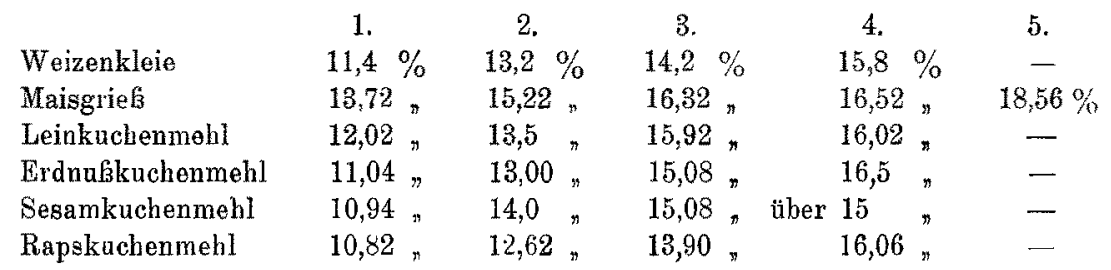

1. Ursprünglicher Wassergehalt, bei 2 . starke, bei 3 . sehr starke, bei 4 . und 5 . (mit Ausnahme von Weizenkleie mit $15,8 \%$ Wasser) geringe Vermehrung.

Das Wasserbedürfnis der einzelnen Arten ist dabei ein verschiedenes. Bei 19,2 bis $21,5 \%$ entwickelten sich von den angewandten Milben nur Glycophagus spinipes befriedigend, während Tyroglyphus farinae und eine Torsoneumart eingingen. Infolge der Milben-Entwickelung nimmt der Wassergehalt der befallenen Stoffe zu, da der Wassergebalt der Milben, wie Maurizio nachwies, $64-68 \%$ beträgt. Diese Steigerung des Wassergehaltes hat alsdann eine Entwickelung von Pilzen zur Folge, die wiederum in kurzer Zeit ein Absterben der Milben nach sich zieht. Unter Milben treten auch manchmal Epidemien auf, so eine, die sich durch Schmierigwerden der Gefäßwände infolge Durchfalls äußert. Eine andere bewirkt ein Eintrocknen der Milben, diese haften an den Wänden und sterben ab. Endlich ist noch eine Pilzkrankheit zu erwähnen, wobei Milben und ihr Substrat gleichzeitig verschimmeln. Allem Anschein nach ist die Pilzkrankheit die primäre, das Verschimmeln des Nahrungs. mittels die sekundäre Erscheinung. Die Milben sind Träger gewisser für Tiere und Menschen ungefährlicher Pilze, wie Rhizopus, Penicillium und anderer, aber auch pathogener und für die Milben gefährlicher Arten von Aspergillus. Das Mißtrauen, welches man überall feuchten und verschimmelten Futtermitteln entgegenbringt, ist vollständig berechtigt.

Eisermann ${ }^{2}$ ) ist der Ansicht, daß Futtermittel, welche Milben enthalten, nur in gedämpftem oder gekochtem Zustande verwendet werden dürfen, da sie meist bei den damit gefütterten Tieren Verdauungsstörungen, auch Hautausschläge hervorrufen. Lindner ${ }^{3}$ ) erwähnt, daß Arbeiter, die andauernd mit einer mit Milben befallenen Gerste in Berührung kamen, sämtlich erkrankten. Ludwig4) berichtet über einen Fall, wo eine ganze Familie, in deren Wohnung Milben in kolossaler Menge lebten, erkrankte.

1) Zentralbl. f. Bakteriol, u. Parasitenkunde, II. Abt., 1906, 15, 606.

2) Eisermann, Getreide und Hülsenfrüchte. Berlin 1895, II, 412.

8) Wochenschr. f. Brauerei $1903,50,548$.

) Ludwig, Die Milbenplage der Wohnungen, ihre Entstehung und Belzämpfung. Leipzig $1904,8.6$. 
Von den verschiedenen Desinfektionsmitteln bewährte sich nach Maurizio am besten schweflige Säure. Luft mit einem Gehalt von $0,2 \mathrm{~g}$ schwefliger Säure in 11 genügte, um Milben samt Brut abzutöten. Von Schwefelkoblenstoff war $0,76 \mathrm{~g}$ für 11 erforderlich. Anilin, Chloroform, Formaldehyd erwiesen sich als sehr unwirksam.

Das beste und einfachste Vertilgungsmittel ist Hitze. Nach Lindner ${ }^{1}$ ) genügt eine Temperatur von $50^{\circ}$, um Milben wie auch ihre Brut in kurzer Zeit abzutöten. Maurizio konnte dies bestätigen; einstündiges Erhitzen auf $50-53^{0}$ war ausreichend.

Zum Vertreiben der im Getreide manchmal auftretenden Kornkäfer (Schmalund Rüsselkäfer) hat sich nach Hoffmann ${ }^{2}$ ) schweflige Säure nicht bewährt.

Wir möchten nun noch kurz die Art der Herstellung der Graupen erwähnen. Im Jahre 1912 erschien im Organ des Verbandes Deutscher Müller ${ }^{3}$ ) eine Reihe von Aufsätzen, hetitelt: Einrichtung einer automatischen Graupenmühle von 25000 Kilo Tagesproduktion. Die darin gegebene Beschreibung ist insofern interessant, weil von der Verwendung von schwefliger Säure Abstand genommen wird. Es heißt darin folgendermaßen: „Beim Aufbringen der Gerste auf den Speicher findet schon eine Vorreinigung statt. Durch einen großen Rost, welcher Holzstückchen usw. zurückhält, gelangt die Gerste zu einem Warehouseseparator, wo eine kräftige Aspiration stattfindet, passiert sodann 2 grob gelochte Siebe, von denen das erste alle größeren Verunreinigungen, das zweite etwa vorhandene Körner von Mais, Erbsen, Wicken usw. ausscheidet. Darauf folgen 2 feinere Siebe zur Entfernung von Unkrautsamen, Sand usw.; Trespe, Spreu, ausgewachsene und angefressene Körner werden beim Auslauf aus dem Sieb mittels eines starken Iuftstromes entfernt. Beim Verarbeiten der Gerste in der Mühle erfolgt eine nochmalige Reinigung in derselben Art und Weise, nur mit dem Unterschiede, daß ein Magnetapparat eingeschaltet wird, um etwa vorhandene Eisenteile zu entfernen. Die Gerste gelangt alsdann zu 2 Flachsichtern, welche sie in 2 Größen scbeidet, und kommt von dort zu der Doppelschälmaschine. Diese Maschine ist auch während des Betriebes zu regulieren um je nach Wunsch eine stärkere oder geringere Pressung auf das Schälgut auszü̈ben. Die Ausschüttung des geschälten und die Zufuhr des Rohmaterials, die früher sehr viel Schwierigkeiten machte, erfolgt heute automatisch durch die Maschine. Bei ununterbrochenem Betriebe der Schälmaschine entwickelt sich allmählich viel Wärme, und es ist daher eine gute Aspiration notwendig, um diese wie auch den entstandenen Staub abzusaugen. Geschieht dies nicht, so findet leicht ein Verschmieren oder Verbrennen des Schälgutes statt. Von der Schälmaschine kommt das Produkt in 2 Hülsenseparatoren, die es vermittels eines stark wirkenden Luftstromes von allen Hülsen und anhaftendem Staub befreien und es alsdann 2 Bürstmaschinen zuführen, die ebenfalls kräftig aspiriert sind und namentlich den Spalt, wo die Schälmaschine nicht einsetzt, säubern. Während nun früher die geschälte Gerste durch Schleifen in die gewünschte Perlform übergeführt wurde, was namentlich bei ganz feinen Körnern einen großen Materialverlust in Form von Mehl nit sich brachte, ist dieser Übelstand seit Einführung der Spaltmaschine fast vollständig behoben. Das gereinigte Schälgut gelangt also zur Spaltmaschine, wo die Körner je nach der Einstellung der Maschine in 2, 3, 4 oder mehr Stücke zerschnitten werden. Um die sich entwickelnde Wärme sowie Staub abzusaugen,

1) Lindner, Mikroskop. Betriebskontrolle im Gärungsgewerbe, 1905, S. 90.

3) Hoffmann, Das Versuchskornhaus. Berlin 1904, S. 184.

3) Der Müller 1904, 34, 659, 683, 711, 733 . 
ist die Maschine an die Zentralaspiration angescblossen. Nachdem die zerschnittenen Körner einen Flachsichter passiert haben, um kleinere Bruchstücke und Staub zu entfernen, werden sie in einem Rollgang rund geschliffen, darauf in einer Bürstmaschine gereinigt und schließlich der Poliermaschine zugefübrt und hier fertiggestellt. In den* jenigen Fabriken, in welchen man schweflige Säure verwendet, wird die rohe Gerste, wie auch hier angegeben, gereinigt, geschält und alsdann mit schwefliger Säure behandelt".

Durch die schweflige Säure soll angeblich eine Sterilisation herbeigeführt werden. Wie sich aus einer Arbeit von Robert Kochl) ergibt, tötet schon eine geringe Menge schwefliger Säure vegetative Formen von Bakterien. Luft mit einem Gehalt von 0,986 Vol. ${ }^{0} \%$ schwefliger Säure tötet feuchte Bakterien in 2 Minuten, an Fäden angetrocknete in 20 Minuten. Anders verhält es sich jedoch mit Sporen. Luft mit 1 Vol.- $\% \mathrm{SO}_{2}$ war bei 3-tägiger Einwirkung ohne jeden Einfluß. Wurde der Gehalt an schwefliger Säure auf $6,13 \mathrm{Vol}_{.}-\%$ erhöht, die Versuchsdauer auf 4 Tage ausgedehnt, in welcher Zeit der Gehalt an schwefliger Säure auf $3 \mathrm{Vol}-\mathrm{O}_{0}$ herabging, so war das Ergebnis ebenfalls negativ.

Koch wandte darauf schweflige Säure in wässeriger Lösung an. Eine derarlige Lösung mit 11,436 Gew.-\% schwefliger Säure verursachte bei 24-stündiger Einwirkung nur eine geringe Verzögerung im Auskeimen der Sporen, aber keine Abtötung. Im Getreide kommen nun sowohl sporenbildende Bakterien wie auch Sporen von Pilzen vor und um so mehr, je unreiner dasselbe ist. Erstere gehören der Gruppe der Kartoffelbacillen an, die uns ja hinreichend bekannt sind wegen ihrer Fähigkeit, fadenziehendes Brot zu bilden. Wir haben nun sowohl rohe russische Gerste wie auch gereinigte, geschälte und von letzterer sowohl geschwefelte wie ungeschwefelte und auch Graupen auf Sporen von Bakterien und Pilzen untersucht und stets solche gefunden. Die Untersuchungen führten wir nach dem Verfahren von Hoffmann ${ }^{2}$ ) aus. Je nach dem vermuteten Keimgehalt wurden 5, 10, 15 oder $20 \mathrm{~g}$ in 100,250 oder $500 \mathrm{ccm}$ steriles Wasser gegeben, eine halbe Stunde unter Umschütteln stehen gelassen und zur Bestimmung der Keimzahl entsprechende Mengen ausgesät. Zwecks Ermittelung der hitzebeständigen Bakterien wurden einige ccm der Flüssigkeit in einem sterilen Gefäß eine halbe Stunde auf $75^{\circ}$ erhitzt, abgekühlt und $1 \mathrm{ccm}$ ausgesät. In beiden Fällen wurde der gewöhnliche bei Wasseruntersuchungen übliche Nährboden verwendet. Zur Feststellung der Pilzkeime wurden 0,5-1 ccm in Pflaumengelatine ausgesät. Zur Ermittelung der Keimzahl haben wir auch noch Versuche mit einem

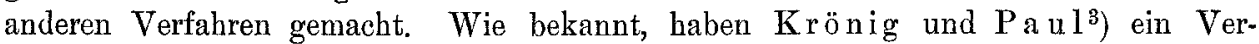
fahren bekannt gegeben zur Ermittelung des Wertes von Desinfektionsmitteln. Sie verwenden dabei Granaten, an welche die zur Prüfung dienenden Keime angetrocknet werden. Wir gingen nun von der Ansicht aus, daß durch Schütteln mit Granaten vielleicht noch ein besseres Ergebnis erzielt werden könnte, indem die scharfen Kanten der Granaten beim Schütteln ein besseres Loslösen der Keime bewirken würden. Um hierüber Aufscbluß zu erlangen, wurden in $100 \mathrm{~cm}$ Wasser $20 \mathrm{~g}$ gereinigte Granaten gegeben, das Ganze sterilisiert, Graupen hinzugefügt, 2, 3, 4 Minuten geschüttelt und jedesmal entsprechende Mengen ausgesät. Es ergab sich zunächst, daß ein 3 Minuten langes Schütteln genügte, um alle Keime loszulösen. Die Ergebnisse waren jedoch.

1) Mitteilungen a. d. Kaiserl. Ges.-Amte 1881, 1, 234.

2) Hof fman n, Das Versuchskornhaus. Berlin 1904, S. 203.

3) Zeitschr. f. Hygiene u. Infektionskrankh. 1897, 25, 1. 
im Vergleich zu denjenigen, die das Hoffmann'sche Verfahren ergab, von einzelnen Fällen abgesehen, nicht besser. Es wurde daher fernerhin nur das Hoffmann'sche Verfahren angewandt. Wir möchten jedoch nicht verfehlen darauf hinzuweisen, daß die Verwendung von Granaten in besonderen Fällen von Vorteil sein kann, z. B. bei der bakteriologischen Untersuchung von Mehl und ähnlichen Stoffen. Beim Schütteln mit Wasser auftretende Klümpchen werden durch die Granaten zerteilt.

Tabelle 1.

Russische Gerste.

\begin{tabular}{r|r|r|r|r|r|r|r}
\hline \multirow{2}{*}{ No } & \multicolumn{3}{|c|}{ Gesamtkeimzahl } & \multicolumn{2}{|c|}{$\begin{array}{c}\text { Hitzebeständige } \\
\text { Bakterien }\end{array}$} & \multicolumn{2}{|c}{ Pilzkeime } \\
\cline { 2 - 7 } & rohe & gewaschene & Abnahme $\%$ & rohe & gewaschene & rohe & gewaschene \\
\hline 1 & 9028000 & 120400 & 98,70 & 583 & 103 & 3200 & 511 \\
2 & 8298000 & 161000 & 98,10 & 1500 & 70 & 1480 & 555 \\
3 & 1103000 & 123800 & 88,88 & 1520 & 44 & 4880 & 466 \\
4 & 2210000 & 127100 & 94,30 & 1360 & 244 & 7600 & 927 \\
5 & 900000 & 108000 & 88,00 & 400 & 180 & 4200 & 640 \\
6 & 1480000 & 98000 & 93,40 & 580 & 210 & 1800 & 180 \\
7 & 1940000 & 110000 & 93,30 & 720 & 140 & 3300 & 320 \\
8 & 2054000 & 85800 & 9580 & 1230 & 220 & 2200 & 110 \\
9 & 7280000 & 46000 & 99,40 & 1300 & 85 & 4200 & 120 \\
10 & 673000 & 61000 & 90,60 & 500 & 120 & 650 & 350 \\
11 & 872000 & 87500 & 90,00 & 420 & 80 & 400 & 550 \\
12 & 321000 & 71750 & 77,65 & 250 & 100 & 700 & 500 \\
13 & 2200000 & 349750 & 84,10 & 1020 & 430 & 2000 & 750 \\
14 & 787000 & 111500 & 85,83 & 400 & 220 & 300 & 350 \\
15 & 534000 & 103250 & 80,67 & 3000 & 840 & 500 & 550
\end{tabular}

Tabelle 2.

Geschälte Gerste.

\begin{tabular}{r|r|r|r|r|r|r}
\hline \hline No. & \multicolumn{2}{|c|}{ Gesamtkeimzahl } & \multicolumn{2}{c|}{ Hitzebeständige Bakterien } & \multicolumn{2}{|c}{ Pilzkeime } \\
\cline { 2 - 7 } & ungeschwefelt & geschwefelt & ungeschwefelt & geschwefelt & ungeschwefelt & geschwefelt \\
\hline 1 & 25000 & 450 & 60 & 30 & 520 & 42 \\
2 & 31000 & 100 & 120 & 50 & 150 & 25 \\
3 & 85600 & 250 & 280 & 20 & 400 & 51 \\
4 & 19300 & 350 & 320 & 35 & 460 & 270 \\
5 & 59400 & 375 & 160 & 55 & 60 & 40 \\
6 & 31400 & 425 & 80 & 110 & 120 & 50 \\
7 & 20200 & 1700 & 1280 & 300 & 210 & 60 \\
8 & 16300 & 325 & 100 & 55 & 120 & 20 \\
9 & 60000 & 2680 & 140 & 180 & 500 & 60 \\
10 & 32800 & 198 & 200 & 120 & 1250 & 150 \\
11 & 55000 & 170 & 120 & 100 & 5920 & 455 \\
12 & 4600 & 150 & 90 & 115 & 465 & 265 \\
13 & 15000 & 120 & 160 & 35 & 2460 & 975 \\
14 & 19800 & 325 & 80 & 50 & 70 & 25 \\
15 & 50050 & 840 & 200 & 180 & 30 & 10 \\
16 & 23750 & 230 & 100 & 90 & 40 & 20 \\
17 & 22250 & 360 & 520 & 230 & 20 & 40 \\
18 & 65350 & 1240 & 200 & 40 & 120 & 80 \\
19 & 36300 & 410 & 160 & 80 & 60 & 70 \\
20 & 44500 & 280 & 200 & 50 & 40 & 60
\end{tabular}


Bei 15 Proben ungereinigter russischer Gerste (Tabelle 1) betrug der Gehalt an Sporen von Bakterien in 7 Fällen über 1000, in 8 Fällen 400 bis 720 , in 1 Falle 250 in $1 \mathrm{~g}$. In letzterem Falle war die Gesamtkeimzahl niedrig, nämlich 321000. Bei den meisten Proben war sie beträchtlich höher, in 9 Fällen zwischen 1103000 und 9028000. Die Anzahl der Pilzsporen betrug in 5 Fällen unter 1000, bei den übrigen war sie bedeutend böher, nämlich bis 7600 in $1 \mathrm{~g}$. In geschälter Gerste Tabelle 2) schwankte der Gehalt an hitzebeständigen Bakterien zwischen 60 und 1280, der Gehalt an Pilzsporen zwischen 30 und 5920, in geschwefelter Gerste (Tabelle 2) zwischen 30 und 300 bezw. 10 und 97 in $1 \mathrm{~g}$. Von Graupen (Tabelle 3) haben wir in derselben Weise 44 Proben untersucht. Bei 11 Proben feiner Perlgraupen schwankte der Gehalt an hitzebeständigen Bakterien zwischen 20 und 803, an Pilzsporen zwischen 40 und 180, bei 16 Proben mittelfeiner Graupen der Gehalt an Sporen zwischen 20 und 210, an Pilzsporen zwischen 20 und 450 in $1 \mathrm{~g}$; bei 17 Proben grober Graupen betrug der Gehalt an Sporen zwischen 15 und 320 , an Pilzsporen zwischen 30 und 630 in $1 \mathrm{~g}$.

Aus diesen Untersuchungen ergibt sich also, daß wir in der Gerste und deren Produkten stets mit der Anwesenheit von Sporen von Bakterien und Pilzen zu rechnen haben. Erstere werden nach den Versuchen von Rob. Koch durch schweflige Säure nicht abgetötet. Bezüglich der letzteren waren noch Untersuchungen anzustellen. Wenn man bedenkt, wie widerstandsfähig Pilze gegen Säure sind, so ist kaum anzunehmen, daß die Sporen derselben durch schweflige Säure abgetötet werden. Dieses muß man auch aus der Tatsache schließen, daß in den untersuchten Graupenproben, die sämulich geschwefelt waren, stets Pilzkeime gefunden wurden. Hiergegen könnte allerdings der Einwand erhoben werden, daß sie erst nachträglich in die Graupen gelangt seien. Wir haben daher 12 Proben geschälter Gerste untersucht (Tabelle 4), dieselben mit schwefliger Säure behandelt und ebenso von derselben Gerste geschwefelte Proben aus der Fabrik erhalten. Die Behandlung nit sehwefliger Säure wurde folgendermaßen durchgeführt: $20 \mathrm{~g}$ geschälter Graupen wurden mit einer Lösung von schwefliger Säure übergossen, welche 4,95 Gew.- $\%$ schweflige Säure enthielt. Obgleich, wie bereits angeführt, Rob. Koch schon nachgewiesen hat, daß sehr geringe Mengen von schwefliger Säure genügen, um vegetative Formen von Bakterien abzutöten, wurde eine so stark konzentrierte Säure angewandt, um ihren Einfluß auf Sporen von Bakterien und Pilzen zu ermitteln. Die Einwirkung dauerte 15 Minuten, alsdann wurde die schweflige Säure abgegossen, mit sterilem Wasser mehrmals nachgewaschen, $100 \mathrm{ccm}$ steriles Wasser hinzugegeben, umgeschüttelt, die noch vorhandene schweflige Säure durch eine Kaliumpermanganatlösung oxydiert und die freie Säure durch eine sterile Lauge neutralisiert. Das Ergebnis war in manchen Fällen fast dasselbe, stets blieben eine Anzabl Pilzsporen vorhanden. Eine Abnahme in den geschwefelten Proben (Tabelle 4) ist zwar nicht zu verkennen, man muß aber andererseits bedenken, daß bei der Art der Versuche durch das Stehenlassen mit der schwefligen Säure und das nachherige Waschen der geschwefelten Proben mit sterilem Wasser zwecks Entfernung der schwefligen Säure Pilzsporen mit fortgeschwemmt werden.

Der Umstand nun, daß durch schweflige Säure keine vollständige Sterilisation zu erzielen ist, ließ es wünschenswert erscheinen, festzustellen, wieweit man durch die übrigen maschinellen Verfahren imstande ist, das Getreide in bakteriologischer Hinsicht zu reinigen, um so mehr da hierüber bisber keine Untersuchungen vorliegen. 


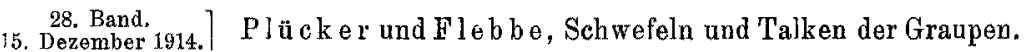

Trabelle 3.

Graupen.

\begin{tabular}{|c|c|c|c|c|c|c|c|}
\hline No. & Art der Graupen & $\begin{array}{c}\text { Talkum- } \\
\text { gehalt } \\
\%\end{array}$ & $\begin{array}{c}\text { Wasser. } \\
\text { gehalt } \\
0 / 0 \\
\end{array}$ & $\begin{array}{c}\text { Schweflige } \\
\text { Säure } \\
\% \\
\end{array}$ & $\begin{array}{l}\text { Gesamt- } \\
\text { keim- } \\
\text { gehalt }\end{array}$ & $\begin{array}{c}\text { Hitze* } \\
\text { beständige } \\
\text { Bakterien }\end{array}$ & $\begin{array}{c}\text { Pilz- } \\
\text { keime }\end{array}$ \\
\hline 1. & Feine Perlgraupen & 0,00 & 12,07 & 0,008 & 411 & 71 & 180 \\
\hline 2. & $n$ & 0,00 & 13,46 & 0,017 & 27986 & 803 & 50 \\
\hline 3. & $n$ & 0,00 & 15,06 & 0,01 & 223 & 52 & 40 \\
\hline 4. & $"$ & 0,32 & 12,55 & 0,0084 & 310 & 60 & 80 \\
\hline 5. & " & 0,34 & 13,05 & 0,013 & 720 & 30 & 40 \\
\hline 6. & " & 0,44 & 13,19 & 0,0093 & 200 & 40 & 40 \\
\hline 7. & $n$ & 0,60 & 13,73 & 0,02 & 210 & 20 & 70 \\
\hline 8 & $"$ & 0,76 & 12,52 & 0,009 & 600 & 40 & 80 \\
\hline 9. & \# & 0,78 & 12,04 & 0,025 & 1200 & 250 & 90 \\
\hline 10. & $\pi$ & 0,84 & 12,88 & 0,0087 & 260 & 35 & 120 \\
\hline 11. & $n$ & 0,88 & 12,03 & 0,0074 & 570 & 70 & 110 \\
\hline 12. & Mittelfeine Grauper & 0,00 & 13,17 & 0,012 & 710 & 120 & 150 \\
\hline 13. & $n$ & 0,00 & 13,27 & 0,009 & 4660 & 30 & 60 \\
\hline 14. & $"$ & 0,00 & 15,03 & 0,011 & 1310 & 210 & 30 \\
\hline 15. & $n$ & 0,00 & 16,04 & 0,01 & 540 & 110 & 20 \\
\hline 16. & $n$ & 0,14 & 12,76 & 0,0065 & 520 & 20 & 450 \\
\hline 17. & " & 0,14 & 12,05 & 0,0052 & 110 & 20 & 25 \\
\hline 18. & " & 0,20 & 12,76 & 0,014 & 140 & 50 & 30 \\
\hline 19. & 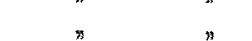 & 0,20 & 12,20 & 0,015 & 220 & 55 & 60 \\
\hline 20. & " & 0,20 & 13.05 & 0,0058 & 260 & 60 & 30 \\
\hline 21. & " & 0,30 & 13,12 & 0,018 & 410 & 40 & 810 \\
\hline 22. & $"$ & 0,34 & 13,07 & 0,013 & 130 & 40 & 30 \\
\hline 23. & 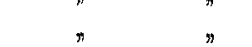 & 0,40 & 14,08 & 0,016 & 190 & 35 & 20 \\
\hline 24. & $n$ & 0,44 & 12,18 & 0,0122 & 200 & 30 & 40 \\
\hline 25. & 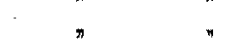 & 0,62 & 13,90 & 0,088 & 320 & 20 & 50 \\
\hline 26. & $n$ & 0,72 & 13,25 & 0,019 & 240 & 25 & 20 \\
\hline 27. & $n$ & 1,02 & 12,37 & 0,010 & 140 & 60 & 40 \\
\hline 28. & Grobe Graupen & 0,00 & 13,22 & 0,015 & 160 & 20 & 40 \\
\hline 29. & 政 & 0,00 & 13,50 & 0,01 & 260 & 40 & 30 \\
\hline 30. & $n$ & 0,18 & 9,54 & 0,008 & 110 & 40 & 30 \\
\hline 31. & " & 0,20 & 12,20 & 0,008 & 1960 & 60 & 40 \\
\hline 32. & " & 0,22 & 12,00 & 0,024 & 170 & 20 & 45 \\
\hline 33. & $n$ & 0,22 & 12,68 & 0,01 & 190 & 20 & 140 \\
\hline 34. & " & 0,34 & 12,56 & 0,028 & 280 & 20 & 180 \\
\hline 35. & , & 0,40 & 12,38 & 0,004 & 300 & 40 & $80^{\circ}$ \\
\hline 36. & $n$ & 0,42 & 12,16 & 0,0044 & 720 & 30 & 630 \\
\hline 37. & , & 0,48 & 12,20 & 0,003 & 580 & 210 & 50 \\
\hline 38. & 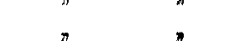 & 0,52 & 11,86 & 0,009 & 310 & 20 & 50 \\
\hline 39. & 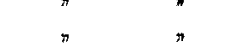 & 0,58 & 12,58 & 0,0015 & 150 & 15 & 30 \\
\hline 40. & $n$ & 0,68 & 12,50 & 0,0087 & 170 & 20 & 80 \\
\hline 41. & ; & 0,80 & 11,21 & 0,018 & 120 & 20 & 40 \\
\hline 42. & 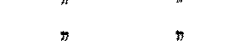 & 1,24 & 11,97 & 0,012 & 160 & 15 & 70 \\
\hline 43. & $"$ & 1,24 & $1 \times, 90$ & 0,011 & 185 & 25 & 30 \\
\hline 44. & $"$ & 1,38 & 11,75 & 0,002 & 680 & 320 & 160 \\
\hline
\end{tabular}


Wir haben uns daher 12 Proben roher Gerste verschafft und von derselben Gerste ebensoviele Proben, die, wie vorhin angegeben, in einem Warehouseseparator von anhaftendem Schmutz und gröberen Verunreinigungen befreit waren (Tabelle 5). Bei 3 der untersuchten Rohgersten betrug der Gehalt an Keimen weit über 1 Million bis 3395000 . Die prozentuale Abnahme durch das Reinigen betrug in 3 Fällen weniger als $50 \%$, nämlich $16,52,35,64$ und $37,14 \%$, in den übrigen Fällen über $50 \%$ bis $91,97 \%$. Bei der durchschnittlich hohen Keimzahl der verwendeten Rohgerste war der Keimgehalt nach dem Reinigen wenigstens immer noch gegen 200000 in $1 \mathrm{~g}$. Da die Keime fast ausschließlich auf der Oberfläche der Körner sitzen, müssen die meisten bei dem nachfolgenden Schälprozeß entfernt werden, und zwar um so vollständiger, je sorgfältiger die Schale von dem Mehlkörper entfernt wird. Untersuchungen von geschälter Gerste haben dies bestätigt. Bei 20 Proben ungeschwefelter, geschälter Gerste (vergl. Tabelle 2) war der Keimgehalt in 1 Falle unter 10000, nämlich 4600 in $1 \mathrm{~g}$, in 4 Fällen $10-20000$, in 3 Fällen 20-30000, in 5 Fällen 30-40000, in weiteren 5 Fällen 40-70000. Wenn man bedenkt, welche großen Mengen von Keimen die Rohgerste durchschnittlich enthält, dann ersieht man hieraus, daß die Abnahme infolge des Schälens eine sehr große ist, und sie würde zweifellos bei den meisten Proben eine noch bedeutendere gewesen sein, wenn das den Körnern anhaftende Mehl, welches beim Schälen entsteht, durch eine Bürstmaschine sorgfältig entfernt worden wäre. Nun ist aber zu bedenken, daß durch das weitere Spalten, Schleifen und Polieren eine noch weitere Entfernung der Keime bewirkt wird. Wir haben 12 Proben fertiger Graupen untersucht (Tabelle 6), welche ohne schweflige Säure hergestellt waren. Der Keimgehalt derselben schwankte zwischen 500 und 49750, in 8 Fällen war er unter 10000, in 3 Fällen unter 1000, nämlich 500, 700 und 850, über 30000 enthielten nur 3 Proben. Nun wird in der Müllerei schon seit langem ein Waschverfahren zum Reinigen des Getreides angewandt; aus solchem Getreide gewonnenes Mehl soll einen ganz besonders reinen $\mathrm{Ge}$ schmack aufweisen. Die Art und Weise des Waschens ist natürlich verschieden. Bei den Maschinen der Firma Luther in Braunschweig setzt man z. B. das Getreide, nachdem es von den gröberen Verunreinigungen befreit worden ist, in einem Abspritzapparat einem Wasserdruck von 3-4 Atmosphären aus. Durch dieses energische Waschen werden alle Schmutzteile, auch beschädigte und angefressene Körner, entfernt. Das gewaschene Getreide kommt darauf in eine Trockenzentrifuge. Die Weiterbehandlung ist von der Art des Weizens usw. abhängig. Handelt es sich z. B. um Hartweizen, so läßt man ihn $4-5$ Stunden liegen, reinigt nach und kann ihn dann sofort vermahlen. Andere Weizenarten oder solche, die durch Regen gelitten haben, verlangen vor dem Mahlen ein Trocknen. In diesen Trockenapparaten, welche durch mehrere Stockwerke gehen und in welche, um das Trocknen zu beschleunigen, Apparate zum Wenden des Getreides eingebaut sind, beträgt die Temperatur je nach der Art des Getreides 50-80 . In dem untersten Teile wird das warme Getreide durch kalte trockene Luft gekühlt, um ein Beschlagen beim Zusammentreffen mit feuchter Luft zu vermeiden. Ein solches Verfahren werden, natürlich nur wenige Keime überstehen. Diese werden aber bei dem nachfolgenden Schälprozeß entfernt werden. Um den Erfolg des Waschens zu ermitteln, haben wir Versuche mit Gerste und Weizen (vergl. Tabelle 1 u. 7) angestellt. Da wir uns aus einer Mühle keine Proben verschaffen konnten, verfuhren wir derartig, daß wir das Getreide mit etwa $20^{\circ}$ warmem Wasser zwischen den Händen rieben, zwischen Filtrierpapier abtrockneten 
Tabelle 4.

Geschälte Gerste, geschwefelt und ungeschwefelt.

\begin{tabular}{|c|c|c|c|c|c|c|}
\hline \multirow{3}{*}{ No. } & \multicolumn{3}{|c|}{ Hitzebeständige Bakterien } & \multicolumn{3}{|c|}{ Pilzkeime } \\
\hline & \multirow{2}{*}{ ungeschwefelt } & \multicolumn{2}{|c|}{ geschwefelt } & \multirow{2}{*}{ ungeschwefelt } & \multicolumn{2}{|c|}{ geschwefelt } \\
\hline & & in der Fabrik & im Laboratorium & & in der Fabrik & im Laberatorium \\
\hline 1 & 60 & 30 & 60 & 520 & 42 & 28 \\
\hline 2 & 120 & 50 & 160 & 150 & 25 & 85 \\
\hline 3 & 280 & 50 & 35 & 400 & 56 & 70 \\
\hline 4 & 320 & 20 & 145 & 460 & 270 & 45 \\
\hline 5 & 160 & 25 & 30 & 60 & 40 & 120 \\
\hline 6 & 80 & 55 & 85 & 120 & 50 & 20 \\
\hline 7 & 200 & 110 & 130 & 210 & 60 & 60 \\
\hline 8 & 1280 & 300 & 60 & 120 & 35 & 50 \\
\hline 9 & 200 & 180 & 120 & 500 & 60 & 130 \\
\hline 10 & 100 & 50 & 80 & 465 & 265 & 210 \\
\hline 11 & 160 & 80 & 100 & 70 & 25 & 40 \\
\hline 12 & 200 & 50 & 120 & 120 & 80 & 70 \\
\hline
\end{tabular}

Tabelle 5.

Rohe und gereinigte Gerste.

\begin{tabular}{r|r|r|r|r|r|r|r}
\hline \hline No. & \multicolumn{3}{|c|}{ Gesamtkeimzahl } & \multicolumn{2}{c|}{$\begin{array}{c}\text { Hitzebeständige } \\
\text { Bakterien }\end{array}$} & \multicolumn{2}{c}{ Pilzkeime } \\
\cline { 2 - 5 } & Rohe & Gereiniate & Abnahme & Rohe & Gereinigte & Rohe & Gereinigte \\
\hline 1 & 3395000 & 948000 & 72,08 & 640 & 230 & 600 & 230 \\
2 & 1367000 & 406950 & 70,23 & 250 & 180 & 500 & 180 \\
3 & 813000 & 397400 & 51,18 & 300 & 200 & 450 & 200 \\
4 & 1278000 & 535000 & 58,14 & 80 & 180 & 100 & 200 \\
5 & 2250000 & 1449500 & 35,64 & 230 & 100 & 180 & 80 \\
6 & 2403000 & 193000 & 91,97 & 100 & 60 & 40 & 100 \\
7 & 1131000 & 562100 & 50,24 & 100 & 20 & 60 & 60 \\
8 & 712100 & 594500 & 16,52 & 1500 & 350 & 100 & 40 \\
9 & 1526000 & 661000 & 56,68 & 30 & 30 & 350 & 150 \\
10 & 2295000 & 712000 & 68,98 & 180 & 50 & 50 & 30 \\
11 & 1512500 & 950500 & 37,14 & 660 & 90 & 100 & 180 \\
12 & 1940000 & 191600 & 90,13 & 40 & 100 & 600 & 90 \\
& & & & & & &
\end{tabular}

Tabelle 6.

Ungeschwefelte Graupen.

\begin{tabular}{c|c|c|c|c|c|c|c}
\hline No. & $\begin{array}{c}\text { Gesamt- } \\
\text { keimzahl }\end{array}$ & $\begin{array}{c}\text { Bitzeheständige } \\
\text { Bakterien }\end{array}$ & Pilzkeime & No. & $\begin{array}{c}\text { Gesamt- } \\
\text { keimzahl }\end{array}$ & $\begin{array}{c}\text { Hitzebeständige } \\
\text { Bakterien }\end{array}$ & Pilzkeime \\
\hline \multirow{2}{*}{1} & 500 & 0 & 0 & 7 & 7500 & 340 & 20 \\
2 & 700 & 20 & 140 & 8 & 9000 & 80 & 60 \\
3 & 850 & 220 & 60 & 9 & 11000 & 40 & 80 \\
4 & 3200 & 20 & 40 & 10 & 35750 & 180 & 20 \\
5 & 5450 & 620 & 20 & 11 & 36800 & 100 & 80 \\
6 & 5610 & 20 & 120 & 12 & 49750 & 100 & 60
\end{tabular}

N. 14. 
Tabelle 7.

Weizen, roher und gewaschener.

\begin{tabular}{|c|c|c|c|c|c|c|c|c|c|c|}
\hline & \multicolumn{3}{|c|}{ Wassergehalt } & \multicolumn{3}{|c|}{ Gesamtkeimzahl } & \multicolumn{2}{|c|}{$\begin{array}{c}\text { Hitzebestandige } \\
\text { Bakterien }\end{array}$} & \multicolumn{2}{|c|}{ Pilzkeime } \\
\hline & $\begin{array}{c}\begin{array}{c}\text { vor dem } \\
\text { Waschen } \\
\% \%\end{array} \\
\end{array}$ & $\begin{array}{c}\text { nach dem } \\
\text { Wasehen } \\
\%_{\%}\end{array}$ & 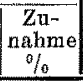 & roher & \begin{tabular}{|c|} 
ge- \\
waschenes
\end{tabular} & $\begin{array}{c}\text { Ab- } \\
\text { nabme } \\
0 \%\end{array}$ & roher & $\begin{array}{c}\text { ge- } \\
\text { waschener }\end{array}$ & Rober & $\begin{array}{c}\text { ge- } \\
\text { waschener }\end{array}$ \\
\hline Landweizen & 9,84 & 19,85 & 10,01 & 7910000 & 15800 & 99,80 & 4280 & 20 & 6200 & 400 \\
\hline Plata & 9,63 & 21,33 & 11,70 & 62962000 & 62000 & 99,80 & 55400 & 78 & 12400 & 160 \\
\hline Manitoba & 9,54 & 21,17 & 11,63 & 1018000 & 48800 & 95,21 & 1832 & 40 & 2100 & 136 \\
\hline Australien & 10,11 & 21,25 & 11,14 & 1108000 & 54000 & 95,10 & 540 & 40 & 13200 & 40 \\
\hline Kansas. & 9,98 & 24,40 & 14,42 & 1332000 & 47000 & 96,50 & 120 & 70 & 4500 & 58 \\
\hline Nordrufland & 10,32 & 20,25 & 9,93 & 1376000 & 60180 & 95,60 & 230 & 110 & 4200 & 60 \\
\hline Ulka. . & 10,28 & 19,98 & 9,70 & 5480000 & 55200 & 99,00 & 420 & 60 & 2800 & 170 \\
\hline
\end{tabular}

und alsdam untersuchten. Nur war in diesem Falle noch eine Bestimmung des Wassergehaltes vorher und nachher notwendig, um eine Berechnung des Keimgehaltes auf denselben Wassergehalt zu ermöglichen. Die Keimabnabme betrug bei Gerste, von der 15 Proben zur Untersuchung kamen, in 6 Fälen unter $90 \%$ bis $77,65 \%$, in den übrigen über $90 \%$ bis $99,4 \%$, bei 7 Weizenproben war das Ergebnis noch besser, es betrug stets über $25 \%$, in 2 Fällen $99,8 \%$. Die Keimzahl sank bei allen Weizenproben weit unter 100000 . Die Höchstzahl war 62000 , die niedrigste 15800, also Zahlen wie wir sie bei geschälter Gerste gefunden haben. Das bessere Ergebnis bei Weizen ist jedenfalls auf die glattere Oberfläche und dadurch bedingte bessere Wirkung des Waschens zurückzuführen.

Welches Verfahren man auch anwenden mag, vollständig entfernen kann man die Keime nicht, auch nicht mit schwefliger Säure, einige werden stets in den Erzeugnissen vorhanden sein; ob dies nun 100 oder 1000 sind, dürfte praktisch ziemlich gleichgültig sein, solange der Wassergehalt so niedrig bleibt, daß keine Vermehrung eintreten kann. Wenn also die Graupenmüller glauben, durch die Behandlung mit schwefliger Säure die Gerste zu sterilisieren, so ist dies nicht zutreffend. Eine solche Behandlung mit schwefliger Säure ist aber auch nach dem Vorhergehenden überflüssig, da ja durch ein rein mechanisches Verfahren die Keime in genügender Weise entfernt werden können. Man muß vielmehr annehmen, daß andere Gründe vorhanden sind, welche die Verwendung von schwefliger Säure empfehlen. Das Verfahren ist bekanntlich durch ein Patent geschützt ${ }^{1}$ ). Die Patentschrift lautet: Verfahren zum Sterilisieren und Bleichen von vorgereinigtem Getreide oder dessen Schälprodukten. Nach einem neueren Patent ${ }^{2}$ ) bebandelt man das Getreide nicht mehr mit schwefliger Säure, sondern einer verdünnten Lösung von Salzen der hydroschwefligen Säure, deren Bleichkraft bekanntlich viel stärker ist. Nun verwendet man in der Graupenmüllerei fast ausschließlich eine russische Gerste, deren Kleberschicht durch Farbstoffe bläulich gefärbt ist, und es dürfte nicht überflüssig sein, die Gründe auseinanderzusetzen, welche die Verwendung dieses Rohproduktes herbeigeführt haben.

Die verarbeitete Gerste kommt zum größten Teil vom echwarzen Meer und den Donauländern. Man baut zwei Sorten, eine bessere, die der deutschen vollständig

1) Deutsches Reichs-Patent Nr. 126202 vom 11. November 1900.

2) Deutsches Reichs-Patent Nr. 167164 vom 6. Februar 1904. 
gleichwertig ist and ein Hektolitergewicht von 67-69 hat, und eine minderwertige, deren Kleberschicht bläulich grau gefärbt ist. Letztere ist leicbt, flachkönig, hat ein Hektolitergewicht von 59-61 und enthält viel verdorbene Körner. Während der Zoll für erștere $400 \mathrm{Mk}$. beträgt, ist er bei letzterer nur $130 \mathrm{Mk}$. Der Preis der besseren Sorte ist ebenso hoch wie derjenige der inländischen oder höchstens un $150 \mathrm{Mk}$. für $10000 \mathrm{~kg}$ billiger, wäbrend sich letztere je nach dem Ernteausfall um $3-400 \mathrm{Mk}$. für $10000 \mathrm{~kg}$ billiger stellt; es besteht also ein ganz erheblicher Preis. unterschied. Russische Futtergerste hat für den Fabrikanten den weiteren Vorteil, dafo sich infolge der Härte des Kornes beim Schleifen weniger Mehl bildet, die Ausbeute an Graupen ist also größer und damit auch der Gewinn, da das Mehl einen geringeren Wert hat. Wie uns von einem Graupenmüller mitgeteilt wurde, eignet sich deutsche Gerste besser zur Graupenfabrikation als russische Futtergerste, erstere ist im Korn mild und weiß and aus ihr hergestellte Graupen sind rein weiß, quellen tadellos beim Kochen und geben einen guten Graupenschleim ab. Graupen aus russischer Gerste sind im Korn hart und quellen beim Kochen weniger gut. Da das Publikum nun die Abnahme der Graupen aus russischer Futtergerste verweigerte,

Tabelle 8.

Wassergehalt russiseher Gerste.

\begin{tabular}{|c|c|c|c|c|c|c|c|c|c|c|c|}
\hline No. & $\begin{array}{c}\text { Vor dem } \\
\text { Waschen } \\
\% \\
\end{array}$ & $\begin{array}{c}\text { Nach dem } \\
\text { Waschen } \\
\%\end{array}$ & $\begin{array}{c}\text { Za. } \\
\text { nahrne } \\
\% \\
\end{array}$ & No. & $\begin{array}{c}\text { Vor dem } \\
\text { Waschen } \\
\% \\
\end{array}$ & $\begin{array}{c}\text { Nach dem } \\
\text { Waschen } \\
\%\end{array}$ & $\begin{array}{c}\mathrm{Zu-} \\
\text { nahme } \\
\%\end{array}$ & No. & $\begin{array}{c}\text { Vor dem } \\
\text { Waschen } \\
\% \\
\end{array}$ & $\begin{array}{c}\text { Nach dem } \\
\text { Waschen } \\
\%\end{array}$ & $\begin{array}{c}\mathrm{Zu}- \\
\text { nahme } \\
\% \\
\end{array}$ \\
\hline 1 & 10,14 & 88 & 12,74 & 6 & 10 & 21,60 & 11,20 & 11 & 9,1 & 19,74 & 10,51 \\
\hline 2 & 11,42 & 22,73 & 11,51 & 7 & 10,72 & 23,04 & 12,22 & 12 & 9,4 & 16,98 & 7,54 \\
\hline 3 & 9,98 & 23,37 & 13,39 & 8 & 9,88 & 24,20 & 14,32 & 13 & 9,63 & 17,34 & 7,71 \\
\hline 4 & 10,20 & 23,47 & 13,27 & 9 & 11,13 & 23,45 & 12,32 & 14 & 9,33 & 18,58 & 9,25 \\
\hline 5 & 11,05 & 22,96 & 11,91 & 10 & 8,91 & 16,29 & 7,38 & 15 & 9,65 & 17,53 & 7,88 \\
\hline
\end{tabular}

griff man zum Bleichen und Talken, was noch den Vorteil hat, dah man, um ein weib und gleichmäbig aussehendes Produkt zu erzielen, die Gerste viel weniger auf den Graupengängen zu bearbeiten braucht. Würde das Bleichen und Talken untersagt werden, so würde sich, wie aus einem Artikel der "Mühle"1) hervorgeht, bald ergeben, „dab die Verwendung der geringen russischen Futtergerste gegenüber schwerer Gerste unvorteilhaft ist, weil diese geringe Futtergerste besonders lange auf den Graupengängen bearbeitet werden müßte, um eine einigermaßen gute Graupe herzustellen, daß aber damn durch das lange kostspielige Bearbeiten und die geringere Ausbeute an Graupen die Vorteile des billigen Einkaufs der Gerste mehr als aufgehoben würden." Das Behandeln mit schwefliger Säure wird nun bewirkt, indem man die Gerste mit Wasser anfeuchtet und alsdann schweflige Säure darauf einwirken läht. Aus unseren Untersuchungen (Tabelle 8) ergibt sich nun, daß der Wassergehalt von 15 Proben roher russischer Gerste sehr niedrig war; bei 12 Proben betrug er bis $10,72 \%$, darunter waren 8 mit weniger als $10 \%$, der höchste war $11,42 \%$. Umgekehrt war der Wassergehalt von 6 Proben geschwefelter Graupen, die frisch aus einer Mühle kamen, bedeutend höher, nämlich 13,22, 13,27, 13,46, $13,5,15,03$ und $15,06 \%$. Es scheint also eine Wasseraufnabme stattzufinden, die nicht überrasehen kann, da, soweit wir erfahren konnten, die Gerste nach dem Be-

1) Die Mühle $1905,42,945$. 
handeln mit schwefliger Säure nicht getrocknet, sondern direkt weiter verarbeitet wird. Dies stimmt überein mit einer Angabe in dem bereits erwähnten Artikel der "Mühle", wonach die Wasseraufnahme so groß ist, daß der Vermahlungsverlust von $11 / 2-2^{1} / 2^{0} / 0$ aufgehoben wird. Von einer Mühle im Elbgebiet heißt es sogar, daß sie es auf diese Weise fertig brachte, eine Ausbeute von über $100 \% \mathrm{zu}$ erzielen, wie durch zollamtlich geprüften Bücherabschluß festgestellt wurde. Es kann den betreffenden Firmen doch nicht unbekannt sein, daß eine solche Beschwerung mit Wasser eine Nahrungsmittelfälschung ist, und man wird gut tun, dieser Seite der Graupenfabrikation ebenfalls Aufmerksamkeit zu schenken.

Durch Einwirkung der schwefligen Säure tritt nun eine Entfärbung ein, sodaß die Gerste hierauf der deutschen vollständig ähnlich sieht. In Wirklichkeit ist dieses Bleichen der Zweck, den man verfolgt, und nicht das Sterilisieren. Würde man das Bleichen unterlassen, so müßte man, um ein verkaufsfertiges Produkt zu gutem Preise losschlagen zu können, die Kleberschicht vollständig abschälen; die Verwendung von schwefliger Säure macht aber ein vollständiges $\Delta b s c h a ̈ l e n ~ d e r$ Klebersehicht überflüssig. Dann ist noch zu bedenken, daß die Behandlung mit schwefliger Säure auch die Verarbeitung eines verdorbenen Getreides gestattet, denn bekanntlich kann man durch Behandeln mit schwefliger Säure verdorbenes Getreide wieder brauchbar machen. Das Verfahren ist ja ebenfalls durch deutsches ReichsPatent ${ }^{1}$ ) geschützt. Durch das Bleichen wird aber der Ware der Anschein einer besseren Beschaffenheit verliehen, es müßte also deklariert werden, wie dies auch bei der Firma Hedrich in Hamburg 1911 einige Zeit geschah; sie brachte auf ihren Erzeugnissen einen Stempel mit dem Aufdruck ,gebleicht" an.

Ist dies nun genügend? Wir stehen auf dem Standpunkt, daf ein Verfahren, welebes auch die Verarbeitung eines verdorbenen Produktes gestattet, unter allen Umständen zu untersagen ist.

Wir kommen nunmehr zum Talken. Nach der Denkschrift der Graupenmüller soll das Talken zweierlei Aufgaben erfüllen, der Zusatz soll

1. als Gleit- und Poliermittel dienen,

2. dadurch, daß er die Poren verstopft, konservierend wirken.

Uns interessiert zunächst das letztere. Eigenschaften, wie sie einem Konservierungsmittel zukommen, fehlen dem Talkum vollständig. Vom theoretisehen Standpunkte aus muß man ihm daher jede Fähigkeit, die Entwickelung von Mikroorganismen zu hemmen, absprechen. Um hierüber Gewißheit zu erlangen, bestimmten wir in 44 Graupen-Proben den Gehalt an Wasser, schwefliger Säure, Talkum, Bakterien und Pilzen. Von den untersuchten Proben waren 11 feine Perlgraupen, 16 mittelfeine und 17 grobe Graupen. (Vgl. Tabelle 3, S. 559.)

\begin{tabular}{|c|c|c|c|}
\hline \multicolumn{4}{|c|}{9 waren frei von Talkum } \\
\hline 3 & & $0,20-0,30 \%$ & alkum \\
\hline 6 & n & $0,30-0,40 \%$ & 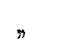 \\
\hline 3 & $n$ & $0,40-0,50 \%$ & $n$ \\
\hline
\end{tabular}

\begin{tabular}{|c|c|c|c|}
\hline & aielter & $0,50-0,60 \%$ & a \\
\hline 2 & $n$ & $0,60-0,70 \%$ & \\
\hline 4 & n & $0,70-0,80 \%$ & \\
\hline 2 & $\pi$ & $0,80 \quad 0,90 \%$ & \\
\hline 4 & $\pi$ & $1,02-1,38 \%$ & \\
\hline
\end{tabular}

Der Gehalt an Wasser war bei 18 Proben unter $12,5^{\circ} \circ$

bei $9, \quad 12,5-13,0 \%$
bei $11, \quad 13,0-13,5 \%$
bei $2 \%$
bei $4, \quad 13,5-14,0 \%$
über $14,0 \%$

1) Deutsches Reichspatent No. 269485 vom 17. November 1912. 
38 Proben, also die große Mehrzahl, enthielten bis $13,5 \%$ Wasser, 18 unter $12,5 \%$. Wenn obige 38 Proben nun auch nicht im Sinne von Schulze ${ }^{1}$ ), der für lagerfeste Gerste einen Wassergehalt von nicht über $12 \%$ verlangt, als lagerfest anzusehen sind, so kommen sie doch dieser Anforderung ziemlich nahe. Der Gebalt an schwefliger Säure war in sämtlichen Proben sehr niedrig, er betrug bei 23 Proben unter $0,01 \%$, der Höchstgehalt war $0,025 \%$. Ein Grund, die Graupen deswegen zu beanstanden, liegt also nicht vor. Bei der Bestimmung der schwefligen Säure machten wir die Beobachtung, daß die Graupen beim Zufügen der Phosphorsäure sich stellenweise rot färbten. Unsere erste Vermutung war natürlich, daß es sich um Eosin handele. Mehrfache Versuche, Eosin nach dem amtlichen Verfahren nachzuweisen, verliefen jedoch negativ. Dagegen ließ sich in Eosingerste auch nach dem Behandeln mit schwefliger Säure Eosin leicht nachweisen, wenn der Farbstoff durch eine Spur Wasserstoffsuperoxyd wieder regeneriert wurde. Ehe wir noch mit der weiteren diesbezüglichen Untersuchung fertig waren, teilte Kollege Jüngermann, Hagen, dem der eine von uns von unseren Beobachtungen Mitteilung gemacht hatte, mit, daß es ihm gelungen sei, mit angesäuertem Alkohol aus der Kleberschicht der russischen Gerste einen roten Farbstoff zu isolieren. Werden Graupen also bei Zusatz von Säure rot, so ist dies ein Beweis für das Vorhandensein russischer Gerste und daß noch Teile der Kleberschicht vorhanden sind. Der Gesamtkeimgehalt war in 4 Fällen über 1000, in 1 Falle sogar 27986 in 1 g. Der Gehalt an Sporen von Bakterien und Pilzen war meistens unter 100. Um nun festzustellen, ob getalkte Graupen haltbarer sind als ungetalkte, verfuhren wir folgendermaßen. In kleine Kästchen aus Zinkblech, etwa je $10 \mathrm{~cm}$ lang und breit und $1,5-2 \mathrm{~cm}$ hoch, deren Boden aus feiner Drahtgaze bestand, brachten wir etwa $100 \mathrm{~g}$ Graupen und stellten dieses Gefäß schräg auf ein zweites ebensolches mit massivem Boden, welches Wasser enthielt. Eine größere Anzahl solcher Behälter, und zwar abwechselnd solche mit Graupen und Wasser, stellten wir unter eine große Glasglocke. Die Graupen mußten hierbei Wasser aufnehmen, und zwar, da sie sich unter denselben Versuchsbedingungen befanden, bei gleicher Größe ziemlich gleichmäßig. Durch Kontrolle des Wassergehaltes wurde dies bestätigt (Tabelle 9). Schwankungen ließen sich hierbei allerdings nicht vermeiden. Es dürfte dies wohl von der Beschaffenheit der Graupen abhängen; solche, welche von weicher Beschaffenheit sind, werden etwas schneller Wasser aufnehmen als solche mit härterer Oberfläche. Ein Besprengen mit Wasser oder Verreiben der Graupen mit Wasser war nicht anwendbar, da hierdurch Talkum, also gerade derjenige Stoff, dessen konservierende Eigenschaften wir prüfen wollten, entfernt wurde. Säntliche Proben, gleichgültig ob der Gehalt an Talkum niedrig oder hoch war, waren nach Ablauf von 7 Tagen stets verschimmelt. Irgendeine hemmende Wirkung des Talkums in bezug auf die Entwickelung der Kleinlebewesen ist also nicht vorhanden. Nun bezieht sich dies ja nur auf Bakterien und Pilze, und man wird vielleicht fragen: Ist es denn mit Milben nicht anders? Wir haben bereits ausgeführt, daß Milben sich am besten bei einem Wassergehalt von $14-16 \%$ entwickeln. Ist dieser vorhanden, so wird das Talkum ibre Vermehrung nicht aufhalten, denn es gibt wohl kaum andere Lebewesen, welche mit so verschiedenartigen Stoffen vorlieb nehmen, wie gerade Milben. Sie nähren sich von Küchenabfällen jeder Art, von dem Kleister der Tapeten, von Getreide, Mehl, Roßhaaren, Tabak, Wein, Bier.

1) Wochenschr. f. Brauerei 1904, 21, 27. 
Tabelle 9.

Wasserafnahme von Graupen aus feuchter Luft.

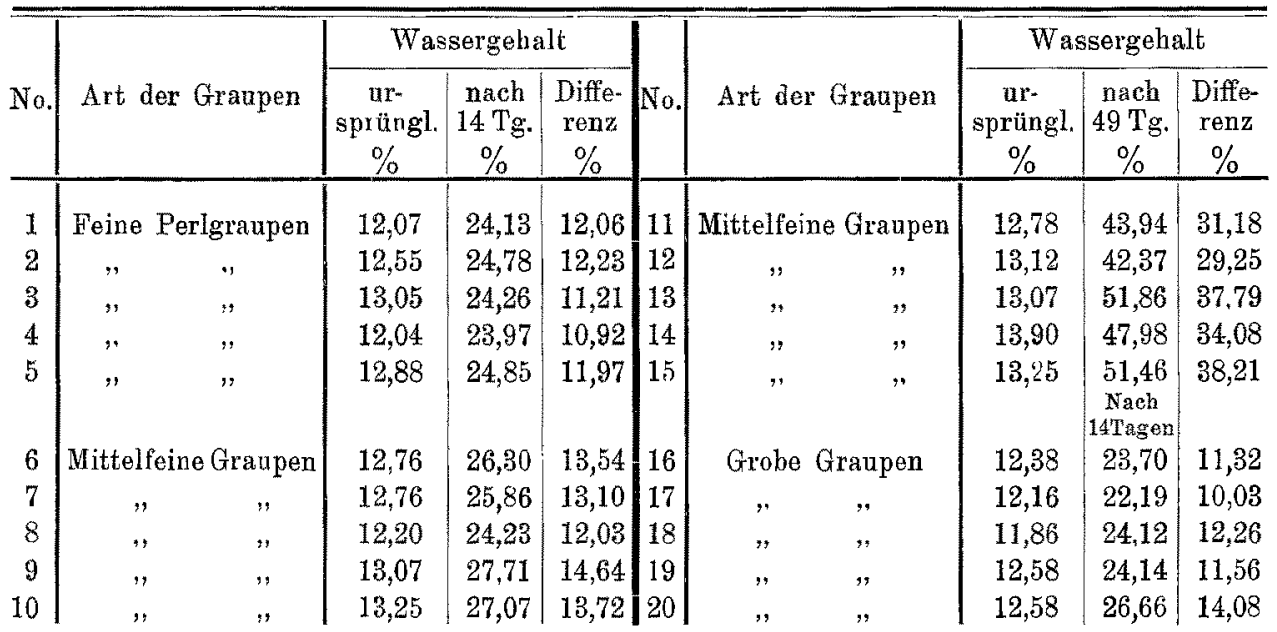

Trouessart ${ }^{1}$ ) erwähnt einen Fall, wo Käsemilben in einer Fabrik nicht weniger als 10000 Büchsen Schubwichse verdarben; als man die Büchsen öffnete, fand man die schwarze Masse fast ganz in graues Pulver verwandelt, das aus unzähligen Milben bestand. Die Büchsen hatten vorher zum Teil vegetabilisches Öl, zum Teil Sirup enthalten, Stoffe, welche die Milben anlockten. Obwohl die Schuhwichse in $1 \mathrm{~kg}$ $50 \mathrm{mg}$ Quecksilberchlorid enthielt, hatten sich die Milben so stark vermehrt. Daß solche Tiere sich durch etwas Talkum nicht abhalten lassen, dürfte ohne weiteres zugegeben werden. Wenn demgegenüber Graupenmüller vor Gericht erklären, daß getalkte Graupen sich besser gebalten haben als ungetalkte, so dürfte die Erklärung hierfür darin zu suchen sein, daß der Wassergehalt der ungetalkten Proben zufällig. ein ziemlich hoher war, höher jedenfalls wie derjenige der getalkten, und daß infolge dieses Umstandes sich bei ersteren in kurzer Zeit Pilze entwickelten, während die anderen infolge des geringen Wassergehaltes ein normales Aussehen bebielten. Eigentümlich ist, daß die Fabrikanten bei Graupen ein Konservierungsmittel für notwendig erachten, und gerade schweflige Säure und Talkum, während dies bei Mehl, was ihnen doch nicht unbekannt sein kann, überflüssig ist. Interessant ist in dieser Beziehung auch eine Mitteilung der Handelskammer in Düsseldorf vom Januar 1913. Es wird darin ein Gutachten von Dr. Wil le cke, Frankfurt, erwähnt, welcher eine Deklaration des Schwefelns und Talkens verlangt hatte und sich dabei auf das Deutsche Nahrungsmittelbuch berief, welches bei Nahrungsmitteln eine Deklaration des Konservierungsmittels verlangt, falls es sich nicht um eins der allgemein gebräuchlichen, wie Kochsalz, Salpeter usw., handelt. Die Handelskammer wandte sich darauf an den Bund Deutscher Nahrungsmittelfabrikanten und Händler, der folgendermaßen antwortete: "Die gefragte Angelegenheit liegt so, daß die im Dẹtschen Nahrungsmittelbuche (2. Aufl. 1909) auf Seite 93 angeführte Behandlung der Graupen mit schwefliger Säure und Talkum allerdings die Festsetzungen, welche sich auf Seite $251 \mathrm{ff}$. befinden,

1) Siehe Ludwig, Die Milbenplage der Wohnungen, ihre Entstehung und Bekämpfung. Leipzig 1904, S. 7. 
ausschließen sollen. Eine anscheinende Unstimmigkeit kann bezüglich dieser beiden Stellen des Nahrungsmittelbuches gefunden werden, wenn man hinsichtlich der Festsetzungen auf Seite $251 \mathrm{ff}$. die Überschrift des genannten Absatzes übersieht. Diese heißt: II. Abschnitt. Dauerwaren. Zu den Dauerwaren in diesem Sinne gehören zweifellos Graupen nicht, vielmehr sind darunter solche aus Fleisch, Obst und Früchten hergestellte zu verstehen. Das Nahrungsmittelbuch geht also von dem Standpunkte aus, daß die Behandlung der Graupen mit schwefliger Säure oder Talkum nicht zu deklarieren ist. Zu dieser Auffassung hinsichtlich der Deklaration sind wir aus dem Grunde gekommen, weil, was namentlich die schweflige Säure anbelangt, allzu geringe Mengen derselben an den Graupen hatten können, um gesundheitsschädlich zu wirken, ganz abgesehen davon, daß die etwa vorhandene schweflige Säure beim Kochen der Graupen ja zum weitaus größten Teil aus der Speise entfernt wird. Auch die geringen Mengen von Talkum, welche an den Körnern haften bleiben, können als gesundheitsschädlich unseres Erachtens nicht angesehen werden. Immerhin nehmen wir keinen Anstand zuzugeben, daß auf Seite 252 Zeile 2 von oben das Wort "Nahrungsmitteln" zu allgemein gefaßt ist und daß statt dieses Wortes zweckmäßiger die Bezeichnung "Dauerwaren" zu setzen wäre."

Man hat bei diesem Gutachten vollständig übersehen, daß die schweflige Säure in erster Linie als Bleichmittel dient. Da nun das Deutsche Nahrungsmittelbuch (Seite 93 ) bei gebleichtem Mehl eine Deklaration verlangt, wird man dies auch bei geschwefelten Graupen als richtig anerkennen müssen.

Wie haltbar gerade Graupen sind, möchten wir noch an einem Beispiel auseinandersetzen. Wir brachten in 4 Exsiccatoren, in deren unterem Teile sich Wasser befand, je eines der erwähnten Zinkkästchen mit Graupen, legten auf das Kästchen 2 Glasstäbe und darauf 2 Filzscheiben. Die Wasseraufnahme war eine außerordentlich langsame, was sich nur durch eine geringe Luftzirkulation durch die Filzscheiben hindurch erklären läßt, und dies bewirkte denn auch, daß die Graupen selbst nach 4 Wochen noch nicht verdorben waren.

Es war nun ganz angebracht zu ermitteln, wie sich Mehl in Vergleich zu Graupen verhält, wenn man es unter denselben Bedingungen in einer feuchten Atmosphäre aufbewahrt. Wir haben je sechs Proben Roggen- und Weizenmehl untersncht, den Gehalt an Wasser, die Gesamtkeimzahl und Pilze ermittelt (Tabelle 10). Was die Zunahme des Wassergehaltes anbetrifft, so ergab sich, dab Mehl am achten Tage entweder ebensoviel oder noch mehr Wasser aufgenommeu hatte wie Graupen in vierzehn Tagen. Das Mehl war nach vier Tagen schon vollständig klumpig, am fünften Tage hatte es bereits einen ganz muffigen Geruch. Die Keimzahl stieg in acht Tagen um mehrere Millionen an. Dies widerspricht allerdings, da der Wassergebalt nach acht Tagen noch keine 30\% betrug, der Arbeit von König, Spieckermann und Kuttenkeuler ${ }^{1}$ ), nach der sich Bakterien erst bei einem Wassergehalt von etwas über 30\% entwickeln sollen. Nach der bereits erwähnten Arbeit von König, Spieckermann und Tillmans ${ }^{2}$ ) vermehren sich allerdings einige Arten schon bei $20 \%$ Wassergehalt, und aus einer vor kurzem erschienenen Arbeit von $\mathrm{Barthe} \mathbf{1}^{3}$ ) ergibt sich, daß manche Bakterien schon bei einem Wassergehalt von $16 \%$ sich vermehren. Es ist aber auch folgendes zu bedenken.

1) Diese Zeitschrift 1906, 11, 178.

2) Diese Zeitschrift 1902, 5, 763.

3) Zeitschr. f. Gärungsphysiologie 1914, 4, 16. 
Die Mehlschicht war etwa $1 \mathrm{~cm}$ hoch, die Wasseraufnahme natïrlich am Boden und auf der Oberfläche am größten. Es ist daher nicht ausgeschlossen, daß stellenweise der Wassergehalt auf $30 \%$ anstieg; also für Bakterien ein günstiger Entwickelungsherd geschaffen wurde. Um den Wassergehalt zu bestimmen, wurde das Mehl jedesmal in einem Mörser gemischt und hiervon Proben genommen.

Tabelle 10.

Wasseraufnahme und Keimgehalt von Mehlen.

1. Roggenmehl.

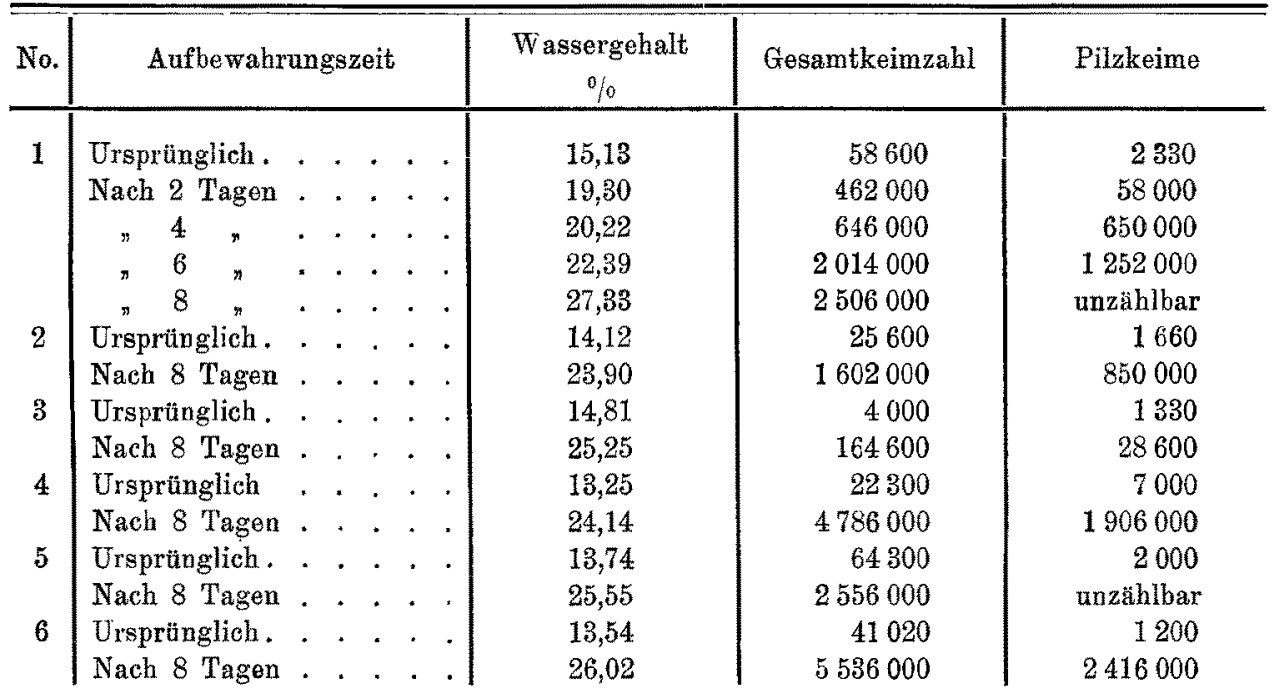

2. Weizenmehl.

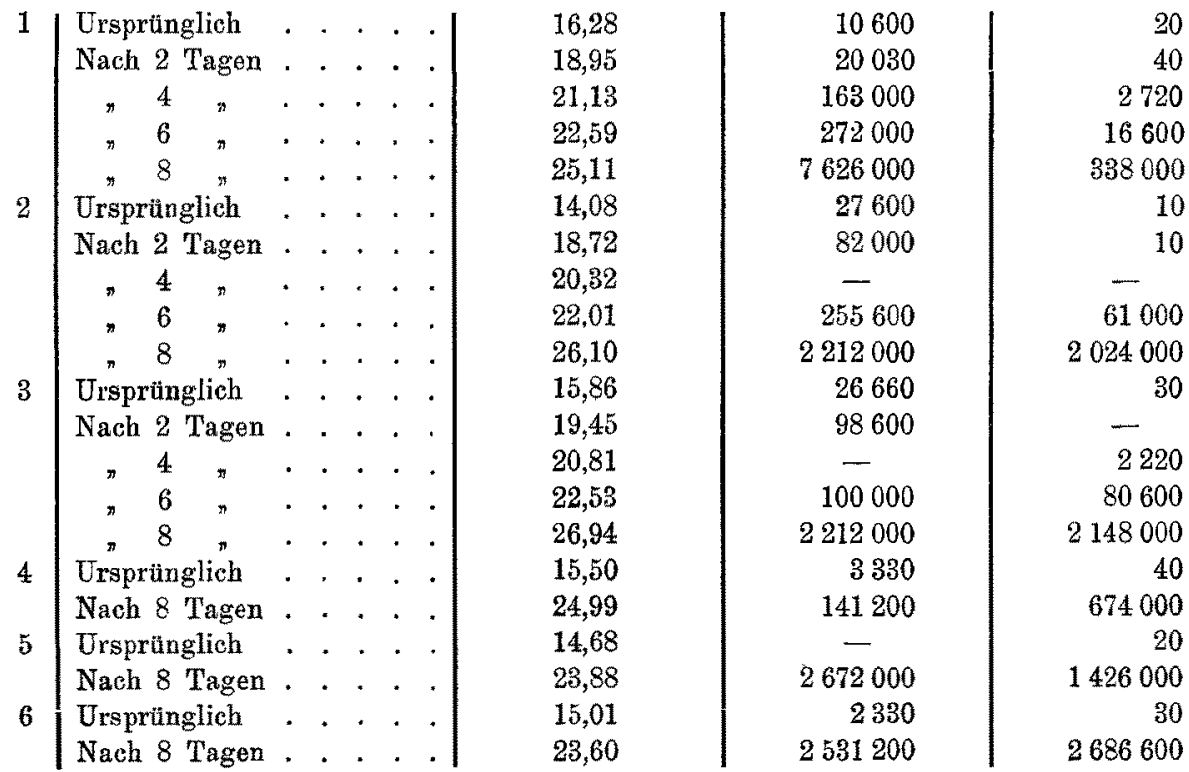


Wenn wir also bei dem leicht verderblichen Mehl ohne Konservierungsmittel auskommen, dann wird ein solches bei Graupen ganz überflüssig sein. Wäre es den Graupenmüllern nur darum zu tun, ein möglichst haltbares Erzeugnis zu erzielen, dann könnten sie dies auf viel einfacherem Wege erreichen. Sie brauchten nur darauf zu achten, daß der Wassergehalt $12 \%$ nicht übersteigt. Ist dies der Fall, und wird die Ware richtig aufbewahrt, dann ist sie unbedingt lagerfest, da ja bei einem solchen Wassergehalt sich weder Bakterien und Pilze noch Milben entwickeln können. In Wirklichkeit bezweckt man mit dem Talken ja auch etwas ganz anderes. In einer Arbeit von Mathes und Müller1) heißt es: „Daß übrigens das Behandeln der Graupen tatsächlich zum Zwecke der Täuschung geschieht, geht aus den Angaben eines Mühlenbesitzers hervor, daß er die Graupen mit Talkum behandle, um ihnen ein etwas besseres Aussehen zu geben. Die Konkurrenz babe damit angefangen, und so sei er jetzt auch dazu gezwungen." Einen besseren Beweis finden wir in den schon erwähnten Aufsätzen aus dem Verbandsorgan Deutscher Müller, es heift darin: „Beim Eintritt in diese Maschine (gemeint ist die Poliermaschine) wird jeder Sorte eine kleine Portion unschädlichen Farbstoffes zugesetzt, die den Körnern im Polierprozesse einen weißlichen Überzug verleiht, wie solches im Handel gewünscht wird." Dieser Farbstoff kann natürlich nur Talkum sein, und damit ist klipp und klar ausgesprochen, was man damit erreichen will. So heißt es in jener Artikelreihe nicht nur an einer, sondern an vier Stellen, ein Druckfehler kann also nicht vorliegen. Merkwürdig ist auch, daß in den ganzen Aufsätzen von einer konservierenden Wirkung dieses Farbstoffes keine Rede ist. Ebenso auffällig ist, daß der Zusatz nicht als zum Polieren notwendig erklärt wird. Der Artikelschreiber scheint also selbst an diese Argumente der Denkschrift der Graupenmüller nicht zu glauben, und da er eine Behandlung mit schwefliger Säure ebenfalls nicht als notwendig erachtet, steht er ja in dieser Beziehung vollständig auf unserem Standpunkt; daß beides entbehrlich ist, beweist ja auch die Tatsache, daß man in Österreich, wo das Schwefeln und Talken verboten ist, ohne solche Mittel auskommt, auch bei uns gibt es noch einige Firmen, welche kein Talkum verwenden. In Dänemark dürfen geschwefelte Graupen nur unter der Bezeichnung ,künstlich gebleichte Gerstengraupen" feilgebalten werden, wenn der Gehalt an schwefliger Säure nicht über $0,3 \%$ beträgt. Das Talkum hat also lediglich den $Z$ weck eines Farbstoffes zu erfüllen. Nun kann man ja einwenden, daß nach unseren vorhergehenden Mitteilungen ein Färben infolge des Bleichens überflüssig sein müsse. Dies dürfte bei einem feinen Rohprodukt unstreitig zutreffen, bei der gewöhnlichen Ware machen sich aber stets Farbunterschiede bemerkbar, die durch Talkum beseitigt werden. Man kann sich hiervon leicht überzeugen, wenn man von getalkter und geschwefelter Ware das Talkum entfernt. Vielleicht ist auch das Schwefeln nicht immer gleichmäßig. Da wir nun bei anderen Nahrungsmitteln ein Färben zulassen, wird man es auch in diesem Falle gestatten müssen, vorausgesetzt, daß es einwandfrei deklariert wird. Da es sich aber um einen Mineralfarbstoff handelt, ist die Festsetzung einer höchstzulässigen Menge geboten.

Wir haben zu diesem Zwecke die Untersuchungsergebnisse von 546 Proben, die in verschiedenen Untersuchungsämtern erhalten wurden, zusammengestellt. Hierbei ergab sich folgendes:

1) Zeitschr. f. öffentl. Chemie 1905, 11, 79. 
34 Proben waren frei von Talkum

113 Proben enthielten bis $0,20 \%$ Talkum

\begin{tabular}{|c|c|c|c|}
\hline 97 & & & $0,20-0,30 \%$ Talkum \\
\hline 61 & & & $0,30-0,40^{\%} / 0$ \\
\hline 8 & 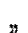 & 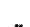 & $0,40-0,50 \%$ \\
\hline
\end{tabular}

334 , also $83,80 \%$ der getalkten Proben, enthielten bis 0,5\%, der Rest, also nur $16,2 \%$, über $0,5 \%$ Talkum. Auf Grund dieses Materials schlagen wir vor, 0,5\% als höchstzulässige Grenze festzusetzen. Vor der Strafkammer, wo dieselbe Frage dem einen von uns kürzlich vorgelegt wurde - die beanstandete Ware enthielt 1,57\% Talkum -, haben wir uns auf denselben Standpunkt gestellt.

Die Ergebnisse unserer Untersuchungen lassen sich folgendermaßen zusammenfassen:

1. Eine Sterilisation der zur Herstellung von Graupen dienenden Gerste ist unnötig, da bei Mehl, welches noch leichter verderblich ist, eine Sterilisation des Rohproduktes ebenfalls unterbleibt. Ebenso wie bei Herstellung von Mehl kann man auch bei der Fabrikation der Graupen Bakterien, Pilze usw. auf maschinellem Wege in genügendem Maße entfermen.

2. Beim Behandeln mit schwefliger Säure wird nur eine unvollständige Sterilisation erreicht. Der Hauptzweck des Verfahrens ist auch nicht die Sterilisation, sondern das Bleichen der in der Kleberschicht der russischen Gerste vorhandenen Farbstoffe, wodurch diese der deutschen ähnlich wird und sich ein vollständiges $A b$ schälen der Kleberschicht erübrigt. Der Ware wird dadurch der Anschein einer besseren Beschaffenheit verliehen. Das Verfahren ist zu untersagen, da es auch die Verarbeitung eines verdorbenen Rohproduktes gestattet.

3. Das Talken der Graupen erhöht in keiner Weise deren Haltbarkeit. Bei gleichem Wassergehalt und gleicher Aufbewahrung verderben getalkte Graupen unabhängig von der Höhe des Talkumgehaltes ebenso schnell wie ungetalkte. Das Talkum erfüllt den Zweek eines Farbstoffes, um der Ware ein gleichmäßig feines Aussehen zu geben, es wird also eine bessere Beschaffenheit vorgetäuscht. Das Talken ist daher zu untersagen oder nur unter der Bedingung zulässig, daß eine einwandfreie Deklaration erfolgt und der Gehalt an Talkum $0,5 \%$ nicht übersteigt.

Vorsitzender: Ich danke Herrn Kollegen Dr. Plücker für seine interessanten Ausführnngen und frage die Herren, ob aus der Versammlung das Wort zu dem Vortrage gewünscht wird.

\section{Diskusion:}

Prof. Härtel: Ich kann mich den Ausführungen des Herrn Referenten nicht anschliefen. Ich habe Gelegenheit gehabt, seit 10 Jahren den Betrieb eiver der größten Graupenmühlen kennen zu lernen. Dort habe ich die Ertahrung gemacht, dak die deutsche Gerste für die Herstellung von Graupen nicht brauchbar ist. Es ist hervorzuheben, daß die deutsche Gerste viel zu weich ist. Es wird kein deutseher Graupenmüller die deutsche Gerste verwenden, sondern russische Gerste, die härter ist. Infolgedessen ist der Vorwurf, daE verdorbene Gerste verwendet wırd, hinfälliy̆. Unsere Mäller können keine verdorbene russische Gterste verwenden, weil die Gerste verzollt werden mub.

Was das Schwefeln anbelangt, so geschieht das nicht der Bakterien, sondern hauptsächlich der Motten halber. Seitdem die Graupenmäller die Gerste schwefeln, haben sie keine Rekla- 
mationen wegen Motten mehr. Dak durch das Schwefeln der Gerste eine besondere Beschaffen heit gegeben wird, ist nicht wahr. Hier ist die robe Gerste und geschwefelte Gen ste.

(Redner zeigt Proben vor:)

Ich habe Proben machen lassen; das Aussehen der Gerste ist fast dasselbe, ob sie geschwefelt wird oder nicht. Ebenso ist ein Unterschied zwischen Graupen aus ungeschwefelter oder gesehwefelter Gerste nicht wahrnehmbar. Der Vorwurf trifft nicht zu, daß durch das Schweteln den Graupen besseres Aussehen gegeben wird.

Was das Talken anbetrifft, so muß ich sagen, ich bin kein Freund davon. Ich habe mich offentlich dagegen gewandt. Ich habe mich äberzeugt, daf die Poliermaschinen in technischer Weise weit vorgeschritten sind und der Betrieb auch mit Gerstenmehl geht. Die Poliermaschinen können aber mit Talkum besser arbeiten. Durch Sichtmaschinen und Schüttelsiebe wird bewirkt, daß das Talkum bis anf eine geringe Menge abgesiebt wird. Infolgedessen kann ich nicht zugeben, wenn Dr. Plä cker sagt, daß die Graupen eine bessere Farbe bekommen. Ieh hebe nochmals hervor, ich selbst bin nicht für das Talken; aber eine bessere Beschaffenheit wird den Graupen nicht gegeben. Die Farbe ist genau gleich.

Regierungsrat Dr. Gä ther: Mit der Frage des Talkens und Schwefelns hat das Kais. Gesundheitsamt sich gleichfalls eingehend befakt und vor einigen Jahren in Gemeinschaft mit dem staatlichen Hygienischen Institut in Hamburg sowie der Kgl. Zentralstelle für offentliche Gesundheitspflege in Dresden in mebreren deutsehen Graupenmählen streng vergleichende Versuche in die Wege geleitet, um festzustellen, ob die Behauptungen zuträfen, nach welchen durch die genannten Verfahren die Haltbarkeit der Graupen ganz wesentlich erhöht werden soll. Es wurden daher nur geschwefelte und nur getalkte Graupen, ferner geschwefelte und getalkte Graupen der verschiedenen Fabrikationsstadien neben unbehandelten Graupen in allen möglichen, praktisch in Betracht kommenden Behältern and Räumen der verschiedensten Art längere Zeit unter mannigfaltigen Bedingungen, bedeckt und unbedeckt, gelagert. Durch Untersuchungen von Zeit zu Zeit wurde festgestellt, wann und in welchem Maße das Ver. dorben der Ware durch die in Betracht kommenden Schädinge, wie Motten, Muben usw, zu beobachten war. Es ist von grofem Interesse, daf diese Versuche - wenn auch auf etwas anderem Wege - praktisch zu dem gleichen Ergebnis gefuhrt baben, wie die Untersuchungen, über die Herr Kollege Plücker berichtet hat. Die geschwefelten und nicht geschwefelten Graupen fielen ungefähr glejch schnell und in gleich starkem Maß3e dem Verderben anheim. Bei den Versuchen des Gesundheitsamtes konnte auferdem festgestellt werden, daß in den deutschen Graupenmühlen ganz überwiegend Futtergerste aus Rußland und den Donanländern, deutsche Gerste mit Rücksicht auf die Preislage und die weiche Beschaffenheit des Korns nur gelegentlich verarbeitet wird. Die ausländischen Gersten enthalten nun vielfach Körner un. gleicher Färbang, und zwar neben gelben viele bläulich und grünlich gefärbte Körner. Diese Färbung beruht auf dem in den äußeren Schichten des Korns eingelagerten natürlichen Pflanzenfarbstoff Anthocyan. Die Schwefelung bezweckt nun in Wirklichlkeit, wie bereits Herr Dr. Plü cker mitgeteilt hat, nichts anderes als eine Bleichung der gefärbten Schichten des Korns. Die Graupenmüblen brauchen die geschwefelte Gerste nicht so oft und stark zu schalen wie die ungeschwefelte Gerste. Es ergibt sich daher, wie aus den dem Gesundheitsamt zugänglichen Beriehten ersichtlich war, für die Mühlen infolge des Schwefelns eine Mehrausbente an Graupen von etwa 10\%. Dieses Ergebnis kann als bedeutender technischer Fortschritt gelten. Da zudem die Menge der in die Graupen gelangenden sohwefligen Säure gering ist und beim Lagern, noch mehr bei der küchenmäfigen Zubereitung der Graupen stark zurückgeht oder ganz verschwindet, da anferdem die in den Graupenmühlen in der Verarbeitung begriffenen ausländischen Gerstensorten bei der Prüfung durch das Gesundheitsamt als völlig gesund und vollwertig befunden wurden, so erschien eine gewisse nachsichtige Beurteilung des Verfahrens nicht ungerechtfertigt.

Anders verhält es sich mit dem Talken der Graupen. Zunächst zeigte es sich auch hier, daß getalkte und nieht getalkte Graupen gleich schnell von Schädlingen befallen wurden, und in einem Falle hatten die am stärksten befallenen Graupen sogar den höchsten Talkumgehalt $(1,6 \%)$. Das Talkum soll - abgesehen von anderen Zwecken - angeblich als Gleitmittel beim Polieren der Graupen dienen, um den in den Trommeln durch das Zusammenkleben der Körner entstehenden Reibungswiderstand zu vermindern, an Kraft zu sparen und ein zu. starkes Erhitzen des Trommelinhaltes zu verhüten. Tatøächlich kann dieser $\mathrm{Z}$ weck auch bei Verwendung von Gerstenmehl erreicht werden. Viele Graupenmühlen sehen daher von einer Behandlung der Graupen mit Talkum ab. Dies geht schon daraus hervor, daki von 168 dem freien Verkehr entnommenen Graupenproben 125 Proben frei von Talkum waren.

Vorsitzender: leh danke Hern Regierungsrat Dr. Günther für seine Mitteilungen. Nunmehr hat das Wort Herr Prof. Härtel.

Prof. Härtel: Ich möchte nur erwidern, daf die Fabrik, wo die Versuche gemacht wurden, nochmals versichert hat, daḱ seit der Zeit, seitdem die Gerste für Graupen gesehwefelt wird, Reklamationen wegen Motten nicht mehr vorkommen, während die Reklamationen früher sehr zahlreich waren. 
Ich hebe nochmals hervor, dak das Schwefeln keinen Vorteil bietet. Die gebleichte Schale wird wieder abgeschält. Es bleiben nur Spuren schwefliger Säure zurück.

Anders ist es mit dem Talkum. Ich bin selbst nicht für das Talken. E's bleibt auch immer etwas Talkum zurück. Es wird jetzt aber überall noch gemacht, und ich hebe hervor, daf, wenn die Sache technisch einwandfrei gemacht wird, die zurückbleibenden Spuren gering sind.

Vorsitzender: Wie Herr Regierungsrat Gunther mitgeteilt hat, lassen sich die Erfahrungen des Kais. Gesundbeitsamtes dahin zusammenfassen, dafi nur ein kleiner Prozentsat/ Gerste talkumiert wird.

Regierungsrat Dr. Günther: Wie schon bemerkt, erwjesen sích von 168 dem freien Verkehr entnummenen Proben 125 als talkumfrej. Bei 67 getalkten Graupen hetrug der Gehalt in 36 Fällen bis $0,5 \%$, in 27 Fällen 0,5 bis $1 \%$ und in 4 Fällen über $1 \%$. Der Höchstgehalt lag bei $1,6 \%$.

Vorsitzender: Ith nehme an, dâf diese Untersuchungen sehr sorgfältig ausgeführt worden sind und diese 125 Proben frei von Talkum waren.

Sie glauben, dafs es möglich ist, das Talkum zu entfernen? Die Müller sollen ohne Talkum arbeiten?

Dr. Plücker: Es ist mir vollständig entgangen, was Herr Kollege Härtel erwähnt hat bezüglich der Erwerbsprodukte, die verarbeitet werden. Daß die Graupenmüller ein einwandfreies Rohprodukt im allgemeinen haben, ist absolut nicht zu leugnen. Es kann nur in einem Falle davon die Rede sein, wo ein derartiges Rohprodukt infolge Feuchtwerdens und Selbsterhitzung gelitten hat. In diesem Falle wird dureh das Behandeln mit schwefliger Säure das Produkt wieder verarbeitungsfähig und verwendbar gemacht, während es sonst nur noch als Viehfutter Verwendung finden kann. Durch die Behandlung mit schwefliger Säure findet tatsächlich ein Bleichen statt, sofern es sich um die gewöhnliche russische Futtergerste handelt.

Die Nuancen in der Farbe bei einzelnen Gerstensorten sind aukerordentlich verschiedenartig. Wenn Sie ein der deutschen gleichwertiges Rohprodukt haben, erzielt man direkt weiße Graupen. Bei der Verarbeitung der gewöhnlichen russischen Gerste machen sich stets bei den Graupen Farbenuntersehiede bemerkbar, auch bei den gebleichten, und diese werden durch das Talkum vollständig behoben.

Prof. Härtel: Dem kann ich nicht zustimmen; denn die fertige Graupe hat, ob sie mit oder ohne l'alkum hergestellt wird, dieselbe Farbe. Ich habe sehr viele Proben gemacht. Wenn eine verdorbene Gerste verwendet wird, ist diese Ware an sich verdorben. Das ist zu beanstanden. Die Graupenmüller müssen gute Gerste verwenden.

Vorsitzender: Ich stelle die Frage, ob noch bei anderen Herren Erfahrungen vorliegen. Dies scheint nicht der Fall zu sem. Wir müssen uns daher begnügen, diese zwei versehiedenen Auffussungen in unserem Bericht wiederzageben. Und es wird sich bei anderer Gelegenheit die Möglichkeit bieten, die Klärung der auseinandergehenden Meinungen herbeizuführen.

Prof. Dr. Härtel: Ich möchte nochmals betonen, daß ich selbst nicht für das Talken bin, während ich das Schwefeln für einen technischen Fortschritt halte,

Vorsitzender: Ich schliebe hiermit die Sitzung, nachdem unsere Tagesordnung erschöpft ist und teile mit, daß morgen vormittag halb neun Uhr unsere geschäftliche Sitzung beginnt. Ich bitte die Herren pünktlich zu erscheinen, weil ein reichhaltiges Programm für diese Sitzung vorliegt. Die öffentliche Sitzung beginnt dann um neun $\mathrm{Uhr}$.

$$
\text { (Schlub der 1. Sitzung } 4 \text { Uhr } 30 \text { Min.). }
$$

Nachmitiags fand eine Besichtigung der Deinhard'schen Kellereien statt.

Abends 8 Uhr fanden sich Teilnehmer und Gäste zu einem gemeinsamen Mahl im Kasino zusammen. 


\title{
2. Sitzung.
}

\author{
Samstag, 23. Mai 1914.
}

In der um $81 / 2$ Uhr beginnenden geschlossenen Sitzung ersucht zunächst

der Vorsitzende, die erforderlichen Wahlen zum Ausschufo vorzunehmen. Satzungsgemäß scheiden aus dem Ausschuß aus die Herren: Rupp, Schumacher, Schwabe, Sendtner, Spaeth und Spieß. Ferner ist als Ersatz für das verstorbene Ausschußmitglied Prof. Dr. N e ufeld-Würzburg eine Neuwahl nötig, sowie eines Stellvertreters des hierfür Gewählten und weiter eines Stellvertreters für Geh. Oberreg-Rat Prof. Dr. von Buchka, dessen bisheriger Stellvertreter ausgeschieden ist.

Direktor Wolfrum schlägt vor, von einer geheimen Abstimmung abzusehen, die satzung:gemä $b$ ausscheidenden Ausschubmitglieder und ibren Stellvertreter durch Zuruf wiederzuwählen und für Herrn Prof. Neufeld dessen Amtsnachfolger Prof. Dr. F. Wirthle-Würzburg, als Stellvertreter für diesen Herm Dr. F. WiedmannRegensburg und als Stellvertreter für Herrn Geh. Oberreg.-Rat von Buchka Herrn Dr. A. Röhrig-Leipzig neuzuwählen.

Dr. S eel wünscht, daß im Ausschuß auch die Militärapotheker vertreten seien; auch seien zu'wenig Vertreter aus Württemberg im Ausschuß.

Dr. Friederich schlägt hierzu Herrn Dr. Seel vor;

Prof. Juckenack bält die Wahl eines Militärapothekers in den Ausschuß nicht für zweckmäßig; wenn eine solche aber doch für nötig erachtet werden sollte, würde als Vertreter wohl zunächst Oberstabsapotheker Dr. Devin-Berlin in Betracht kommen.

Dr. Nottbohm regt die Wahl von Dr. Mezger-Stuttgart an.

Prof. Bömer weist darauf hin, daß Dr. Bujard-Stuttgart schon Ausschußmitglied sei.

Dr. Schwabe schlägt Wiederwahl der satzungsgemäß Ausscheidenden durch Zuruf vor. (Bleibt ohne Widerspruch.)

Der Vorsitzende ersucht die Neuwahlen durch Stimmzettel vorzunehmen.

Er macht weiter Mitteilung über die am 22. Mai stattgebabte Ausschußsitzung, die beschlossen habe, künftig die wichtigsten Ergebnisse dieser Sitzungen den Mitgliedern zur Kenntnis zu bringen,

Es soll von jetzt an nur mehr eine Ausschußsitzung gelegentlich der Hauptversammlung und im Herbst nur eine Vorstandssitzung stattfinden.

Dem Vorstand müssen daher etwas erweiterte Rechte gewäbrt werden.

Er berichtet ferner über den im März 1914 in Berlin stattgehabten Fortbildungskursus, der einen allseitig befriedigenden Verlauf nahm und der vorerst alle zwei Jahre wiederholt werden soll.

Dr. $\mathrm{Ha} B$ regt an, daß zu den Fortbildungskursen nur geprüfte Nahrungsmittelchemiker zugelassen werden sollen.

Geh.-Rat von Buch ka widerspricht dem.

Prof. Juckenack schlägt vor, nur akademisch gebildete Chemiker zuzulassen, womit sich die Herren Geh. Oberreg.-Rat von Buchka und Dr. Haß einverstanden erklären.

Der Vorsitzende bittet um die Wahl des Ortes für die 14. Hauptversammlung 1915; es liege von früher noch eine Einladung nach Cassel vor.

Dr. Paulmann wiederholt diese Einladung herzlichst.

Der Vorsitzende stellt fest, daß Cassel gewählt wurde, und bittet um Vorschläge, welche Referate für Cassel in Aussicht zu nehmen sind. 
Als solche werden von Dr. Murdfield weinhaltige und weinähnliche Getränke, von Dr. von Roehl Obstweine und alkoholfreie Getränke und von Prof. Bömer Fleischpräparate vorgeschlagen.

Die Referenten sollen vom Ausschuß eruannt werden.

Das Ergebnis der Stimmenzählung war die Wahl der Herren Prof. Wirthle, Dr. Wiedmann und Dr. Röhrig.

Im übrigen wurde gegen die Mitteilungen des Vorsitzenden kein Widerspruch erhoben.

\section{Öfentliche Sitzung.}

Der Vorsitzende eröffnet um $91 / 4$ Uhr die öffentliche Sitzung und erteilt dem Geschäftsführer das Wort:

Prof. Mai teilt mit, dab die Kasse im Jahre 1913 einen Ausgleich in Einnahmen und Ausgaben von 4619,80 Mk. zeigt.

Einnahmen:

Vortrag vom Jahre 1912 . . . . 234,29

Einnahmen aus Mitgliederbeiträgen. 4385,51

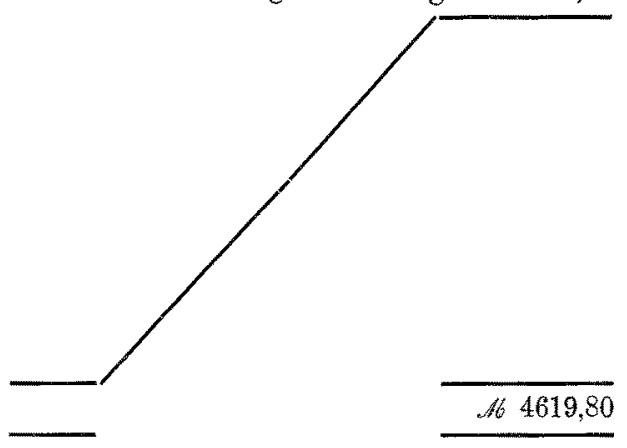

A usgaben:

Scheck-Konto Bay. Hyp.\&Wechselbk. $\quad 200,-$ Versammlungsbericht 1913 . . . . 436,Geschäftsfährer . . . . . . . . $350,-$ Schreibgebühren . . . . . . . . 82,05 Drucksachen . . . . . . . . , 148,80 12. Hauptversammiang . . . . . 139,Reisekosten . . . . . . . . . 1580,70 Porti, T'elegramme . . . . . . . 220,81 Auslagen des Vorsitzenden. . . . 64,45 Kleine Spesen . . . . . . . . 32,70 Khren-Adresse . . . . . . . . 160,Vortrag auf 1914 . . . . . . . 1205,29 4619,80

Das Guthaben auf Scheckkonto bei der Bayerischen Hypotheken- und Wechselbank München hatte Anfang Januar 1914 die Höhe von Mk. 5497,65 erreicht.

Der Vorsitzen de teilt mit, daß die Kassenbücher und Belege von den Herren Schumacher und Spaeth geprüff und richtig befunden wurden; er ersucht, dem Geschäftsführer Entlastung zu erteilen, wogegen kein Widerspruch erhoben wird.

Prof. Mai teilt weiter mit, daß der Verein 2 Fhrenmitglieder und 451 Mitglieder besitzt. Neu eingetreten sind im vergangenen Jahr 12, ausgetreten 7, gestorben 6 Mitglieder; letztere waren die Herren Dr. Hefelmann-Dresden, Dr. Kaute-Berlin, Prof. Dr. Neufeld-Würzburg, Dr. van Noenen-Aachen, Dr. Ohlendorf-Osnabrück und Prof. Dr. Schär-Strabburg i. E.

Der Vorsitzende bittet die Versammlung sich zu Ehren der Verstorbenen von den Sitzen zu erheben.

Dr. Buttenberg schlägt vor, bei den Versammlungen künftig schon am ersten Tag eine Anwesenheitsliste herauszugeben.

Vorsitzender: Ich möchte nun vorschlagen, daß wir am Vormittag alles exledigen, da, wie sich gestern herausgestellt hat, das Frühstück bei der großen Anzahl der Herren sich nicht in einer Stunde erledigen läßt.

Tch möchte nunmehr die beiden neugewählten stellvertretenden Ausschußmitglieder fragen, ob sie gewillt sind, die Wahl anzunehmen. 
Zunächst frage ich Herrn Dr. Wiedmann, ob er die Wahl annimmt.

Dr. Wiedmann: Ich danke vielmals für das Vertrauen, das Sie mir entgegenbringen und nehme die Wahl an.

Darf ich auch Herrn Dr. Röhrig bitten, sich zu äußern?

Dr. Röhrig: Tch danke ebenfalls für das mîr erwiesene Vertrauen und nehme die Wabl an.

Die beiden Kollegen sind also zu stellvertretenden Ausschußmitgliedern gewählt.

Nunmehr hat das Wort Herr Prof. Beythien zu seinem Vortrage.

\section{Die Neuregelung der Nahrungsmittelgesetzgebung}

von

\section{A. Beythien.}

Das zurzeit geltende Nahrungsmittelgesetz vom 14. Mai 1879, welches dem dringenden Wunsche der Bevölkerung und mehrfacher Anregung aus dem Reichstage seine Entstehung verdankte, hat im großen und ganzen seine Aufgabe: Schutz der Konsumenten gegen gesundheitliche und wirtschaftliche Schädigung durch ungeeignete Nahrungsmittel, erfüllt. Die Erfolge der Nahrungsmittelkontrolle, die in kurzer Zeit die gröbsten Falsifikate aus dem Handel verdrängte, sprechen für die Wirksamkeit des Gesetzes, und ihre Vertreter haben daher stets, von einigen kleinen Schönheitsfehlern abgesehen, die Zwekmäßigkeit der gesetzlichen Bestimmungen anerkannt.

Um so lebhaftere Beschwerden wurden dafür aus den Kreisen des Handels und der Industrie erhoben, Beschwerden, die sich zum Teil gegen das Gesetz selbst, zum Teil gegen die Art seiner Handhabung richteten. Die Kleinhändler wiesen immer wieder darauf hin, dab sie fast ausschließlich durch die Kontrolle belästigt würden und daß die Beschränkung der Revisionen auf die Ladengeschäfte eine außerordentlicbe Härte darstelle. Der vom Zentralverbande Deutscher Kaufleute und Gewerbetreibender an den Bundesrat gerichtete Antrag: „Den Beamten der Polizei das Recht einzuräumen, in die Fabriken einzutreten und hier Proben zu entnehmen", gegen welchen berechtigte Einwände wohl kaum erhoben werden können, entspringt dem Wunsche der Kleinhändler, unzulässige Waren von vornherein vom Handel auszuschließen.

Demgegenüber wenden sich die Fabrikanten und zum Teil die Vertreter des Großhandels, deren Warenvertrieb sich über größere Bezirke erstreckt, in erster Linie gegen die "Rechtsunsicherheit", die darin liegt, dab die gleiche Ware nicht von allen Gerichten in gleicher Weise beurteilt wird. Daß dieser Übelstand besteht, läßt sich nicht bestreiten. Wie Sie wissen, verlangen die sächsischen Gerichte bei Eiernudeln einen bestimmten Mindestgehalt an Eiern, während das Landgericht Frankfurt diese Forderung als unberechtigt ansieht. Ein mit Stärkesirup vermischter sog. Tafelhonig ist von der Strafkammer in Dresden als verfälscht beurteilt worden, während die Staatsanwaltschaft in Magdeburg früher - die Angelegenheit liegt 10 Jahre zurück - ein strafrechtliches Einschreiten ablehnte, und ähnliche Beispiele ließen sich zu Dutzenden anführen.

Wenn schon es sich hier weniger um einen bes onderen Mangel der Nahrungsmittelkontrolle als vielmehr un eine allgemeine Folge der Sachverständigen-Vernehmung handelt, der in gleicher Weise bei der Rechtsprechung über Handel mit 